\title{
Zur Element-Stickstoff-Doppelbindung in Kationen cyclischer Bis(amino)-phospha-, -arsa-, -stiba- und -bismutane
}

\author{
M. VeIth*, B. Bertsch und V. HuCH \\ Saarbrücken, Institut für Anorganische Chemie der Universität des Saarlandes
}

Inhaltsübersicht. Bis(amino)silaphospha-, -arsa-, -stiba- und bismaetidine mit positiver Ladung und einem formal nur zweibindigen Element der 5 . Hauptgruppe können aus den entsprechenden Bis(amino)elementchloriden durch Ubertragung des Chloridions auf eine Lewis-Säure wie $\mathrm{AlCl}_{3}, \mathrm{GaCl}_{3}$ oder $\mathrm{InCl}_{3}$ erhalten werden. Röntgenstrukturanalysen an den Verbindungen $\mathrm{Me}_{2} \mathrm{Si}\left(\mathrm{N}^{\mathrm{t}} \mathrm{Bu}\right)_{2} \mathrm{P}^{+} \cdot \mathrm{AlCl}_{4}-(\mathbf{2 a}), \mathrm{Me}_{2} \mathrm{Si}\left(\mathbf{N}^{\mathrm{t}} \mathbf{B u}\right)_{2} \mathrm{Sb}^{+} \mathrm{AlCl}_{4}-(\mathbf{2 c})$ und $\mathrm{Me}_{2} \mathrm{Si}\left(\mathbf{N}^{\mathrm{t}} \mathbf{B u}\right)_{2} \mathrm{Bi}^{+} \mathrm{AlCl}_{4}^{-}$(2d) zeigen, daß die Elektronenarmut am Element $(V)$ durch zwei unterschiedliche Bindungsarten kompensiert wird. Im Falle des Phosphorderivates $2 a$ geschieht der elektronische Ausgleich intramolekular durch die benachbarten Stickstoffatome (Mittelwert $\mathbf{N}-\mathbf{P}^{+}=163,3 \mathrm{pm}$ ), während in dem Antimon(2c) und Bismut-Derivat (2d) die Chlorsubstituenten der $\mathrm{AlCl}_{4}$-Anionen intermolekular das Element(V) absättigen (Mittelwerte: $\mathrm{Cl} \cdots \mathrm{Sb}=305, \mathrm{Cl} \cdots \mathrm{Bi}=309 \mathrm{pm}$ ). Die $\mathbf{N}-\mathrm{Si}-\mathrm{N}$-Gruppierung, die in allen Molekülen als identischer Teil vorhanden ist, kann als Sonde für die Elektronenverteilung im Ring benutzt werden. Die Abstände und Winkel innerhalb dieser Gruppe variieren stark mit der Elektronenakzeptorfähigkeit des Elementes, an das sie gebunden ist. 2a kristallisiert orthorhombisch, Raumgruppe Pnma, $Z=8(a=3023,7(9), b=1001,0(3), c=1414,6(5) \mathrm{pm})$ und $2 \mathrm{c}$ und 2d sind isotyp, ebenfalls orthorhombisch, Raumgruppe Pbca mit $Z=8$ (2e a $=2030,8(8), b=$ $1.193,1(4), c=1777,1(6) \mathrm{pm} ; 2 \mathbf{2}: \mathrm{a}=2025,9(8), b=1198,0(4), \mathrm{c}=1761,3(6) \mathrm{pm})$.

The Element-Nitrogen Double Bond in Cations of Cyclic Bis (amino)phospha-, -arsa-, -stiba-, and bismuthines

Abstract. Bis(amino)sila-phospha, -arsa-, -stiba-, and bismaetidines which bear a positive charge and incorporate a formally two valent element of group $V$ are obtained from the corresponding bis(amino)elementchlorides by transfer of the chloride anion to the Lewis acids $\mathrm{AlCl}_{3}, \mathrm{GaCl}_{3}$, and $\mathrm{InCl}_{3}$. X-ray structure analyses on the compounds $\mathrm{Me}_{2} \mathrm{Si}\left(\mathrm{N}^{\mathrm{t}} \mathrm{Bu}\right)_{2} \mathrm{P}^{+} \mathrm{AlCl}_{4}-(\mathbf{Z a}), \mathrm{Me}_{2} \mathrm{Si}\left(\mathrm{N}^{\mathrm{t}} \mathrm{Bu}\right)_{2} \mathrm{Sb}^{+} \mathrm{AlCl}_{4}^{-}$ (2c), and $\mathrm{Me}_{2} \mathrm{Si}\left(\mathrm{N}^{\mathrm{t}} \mathrm{Bu}\right)_{2} \mathrm{Bi}^{+} \mathrm{AlCl}_{4}{ }^{-}$(Qd) reveal that the electron lack at the element(V) can be compensated by two different bonding mechanisms. In the case of the phosphorus derivative $2 a$ the electronic balance is accomplished by intramolecular backbonding from the neighbouring nitrogen atoms (mean $\mathrm{N}-\mathrm{P}^{+}=\mathbf{1 6 3 . 3} \mathrm{pm}$ ). In the antimony and bismuth derivatives (2e and $2 \mathbf{d}$ ) the chlorine atoms of the $\mathrm{AlCl}_{4}$ anions coordinate to the unsaturated element(V) in an intermolecular manner (mean: $\mathrm{Sb} \cdots \mathrm{Cl}=305, \mathrm{Bi} \cdots \mathrm{Cl}=309 \mathrm{pm}$ ). The $\mathrm{N}-\mathrm{Si}-\mathrm{N}$ group which is identical in all molecules, may be used as a probe for the electronic balance within the ring systems. The bond lengths and angles vary dramatically with respect to the electron acceptor properties of the element to which the group is bonded. $\mathbf{2 a}$ forms orthorhombic crystals, space group Pnma, $Z=8$ ( $a=3023.7(9)$, $b=1001.0(3), c=1414.6(5) \mathrm{pm})$, and $\mathbf{2 e}$ and $2 \mathbf{d}$ are isotypic, again orthorhombie, space group Pbca with $Z=8(\mathbf{2} a=2030.8(8), b=1193.1(4), c=1777.1(6) \mathrm{pm} ; \mathbf{2 d}: \mathrm{a}=2025.9(8), b=1198.0(4)$, $\mathrm{c}=1761.3(6) \mathrm{pm})$. 


\section{Einleitung}

Wie wir in einer vorhergehenden Veröffentlichung [1] zeigen konnten, lassen sich neben dem schon länger bekannten Bis(amino)-phosphorchlorid 1a $[2,3]$ auch die entsprechenden Verbindungen der schweren Homologe des Phosphors 1h, 1e, 1 d problemlos erhalten.

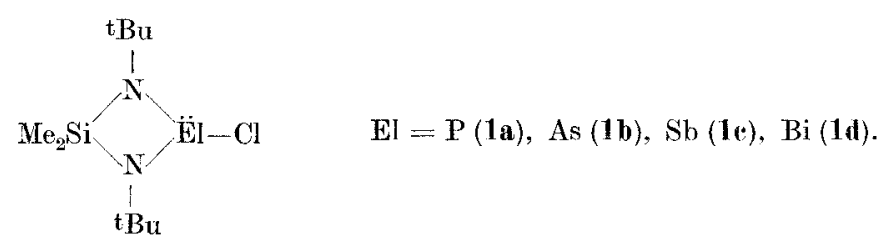

In der vorliegenden Arbeit beschreiben wir die Reaktion dieser Verbindungen des Typs 1 mit Lewis-Säuren wie Aluminiumtrichlorid, Galliumtrichlorid und Indiumtrichlorid (vgl. Fragestellung in Lit. [1]). Bei der Charakterisierung der erhaltenen Produkte stand im Vordergrund, einen Existenznachweis für die erwarteten Kationen zu führen, die isoelektronisch zu den bereits bekannten Bis(amino)germylenen, -stannylenen und -plumbylenen $[4,5]$ sein sollten. Aus diesem Grunde haben wir, neben anderen Methoden, die Röntgenstrukturanalyse verwendet.

\section{Synthese und Eigensehaften von 1,3-Diaza-2-sila-4-elementkation-etidinen}

Bis(amino)phosphorchloride acyclischer [6-10] sowie cyclischer Struktur $[11,12]$ sind häufig mit $\mathrm{AlCl}_{3}$ als Lewis-Säure in die entsprechenden $\left(\mathrm{R}_{2} \mathrm{~N}\right)_{2} \mathrm{P}^{+} \mathrm{AlCl}_{4}^{-}$Ionenpaare umgewandelt worden. Vergleichbare Syntheseversuche an Bis(amino)arsa-, -stiba- und -bismachloriden sind nicht systematisch bzw. nur vereinzelt durchgeführt worden [13]. Galliumtrichlorid bzw. Indiumtrichlorid wurden als Chloridakzeptoren unseres Wissens in diesem Zusammenhang nicht verwendet.

Die cyclischen Bis(amino)elementchloride 1a-1d reagieren mit den Trichloriden des Aluminiums, Galliums und Indiums nach der allgemeinen Gl. (1).

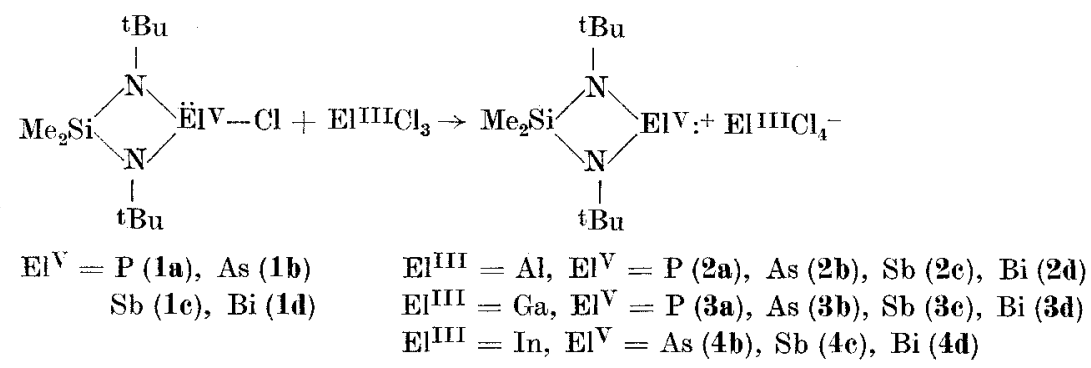

Als Lösungsmittel wird in allen Fällen Benzol verwendet, woraus die meist schwerlöslichen Verbindungen $\mathbf{2 a}-\mathbf{2 c}, \mathbf{3 a}-\mathbf{3 e}$ und $4 \mathbf{b}, \mathbf{4 c}$ ausfallen bzw. auskristallisieren. Die Bismutderivate 2 d, $3 \mathrm{~d}$ und 4d sind wesentlich leichter löslich. Nach kryoskopischer Molmassenbestimmung ist $2 \mathbf{d}$ in Benzol zu einem Dimeren 
assoziiert. In den anderen Fällen findet man nur monomere Moleküle. Die ermittelten Molmassen zeigen, daß in Benzol keine Dissoziation in Kationen und Anionen erfolgt. Es könnten somit Ionenpaare vorliegen oder über Chlorbrücken koordinierte Addukte. Die ${ }^{1} \mathrm{H}-\mathrm{NMR}$-Spektren aller Derivate bestehen aus zwei Singuletts, entsprechend jeweils chemisch gleichwertigen Methylsilyl- und tertButylgruppen. Auf Grund des einfachen Spektrums auf hochsymmetrische Strukturen in den Monomeren oder Dimeren in Lösung zu schließen, ist sehr fragwürdig, da der Ligandenaustausch an den $\mathrm{El}^{\mathrm{v}}$-Atomen sehr schnell gegenüber der NMR-Zeitskala erfolgen kann (vgl. auch Lit. [1]).

Es fällt auf, daß nur das Phosphorderivat 1a mit Indiumtrichlorid nicht zur Reaktion gebracht werden kann: nach Einengen der Lösung verbleibt unumgesetztes $1 \mathrm{a}$ neben $\mathrm{InCl}_{3}$. Dennoch wechselwirken beide Moleküle miteinander, als sich das schwerlösliche $\mathrm{InCl}_{3}$ in Anwesenheit von 1a in Benzol auflöst. Offenbar besteht ein Gleichgewicht, das aber entsprechend Gl. (2) beim Einengen wieder in Richtung der linken Seite verschoben wird.

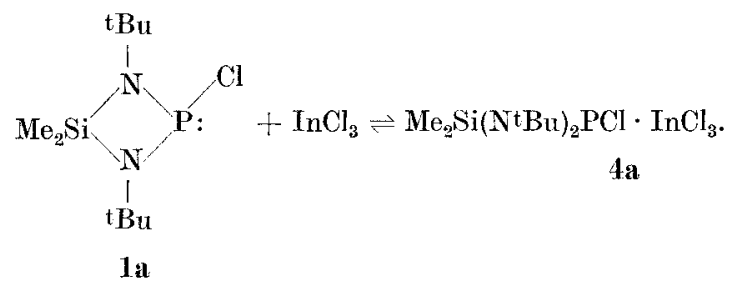

Die Produkte $\mathbf{2 a - 2 d}, \mathbf{3 a - 3 d}$ und $\mathbf{4 b - 4 d}$ sind in kristalliner Form unter Ausschluß von Luft und Feuchtigkeit unbegrenzt haltbar, ebenso lösen sie sich ohne Zersetzung in unpolaren Lösungsmitteln. In Diethylether erfolgt ein Abbau der Verbindung, was offenbar durch irreversiblen Angriff des Sauerstoffs der Ethergruppe an der Lewis-Säure $\mathrm{El}^{\mathrm{III}} \mathrm{Cl}_{3}$ ausgelöst wird.

Tabelle 1 Die energetisch niedrigste Absorptionsbande in den UV-Spektren von $\mathbf{2 b}, \mathbf{3 b}, \mathbf{4 b}$ und 2b, 2e, 2d.

Neben der Wellenlänge $\lambda$ (in $\mathrm{nm}$ ), ist in Klammern die jeweilige Molextinktion angegeben $\left(\mathrm{X}_{2}\right.$ steht für $\left.\mathrm{Me}_{2} \mathrm{Si}\left(\mathrm{N}^{\mathrm{t}} \mathrm{Bu}\right)_{2}\right)$.

Zum Vergleich ist auch die entsprechende Bande in $\mathrm{X}_{2} \mathrm{Sn}$ (5c) aufgeführt [14]

\begin{tabular}{lll}
\hline $\mathrm{X}_{2} \mathrm{As}^{+} \mathrm{AlCl}_{4}^{-}(\mathbf{2 b})$ & $\mathrm{X}_{2} \mathrm{Sb}^{+} \mathrm{AlCl}_{4}^{-}(\mathbf{2} \mathbf{c})$ & $\mathrm{X}_{2} \mathrm{Bi}^{+} \mathrm{AlCl}_{4}^{-}(\mathbf{2 d})$ \\
$\lambda=346(427)$ & $\lambda=364,6(1419)$ & $\lambda=434(1946)$ \\
$\tilde{v}=28248 \mathrm{~cm}^{-1}$ & $\tilde{v}=27434 \mathrm{~cm}^{-1}$ & $\tilde{v}=23041 \mathrm{~cm}^{-1}$ \\
& & \\
$\mathrm{X}_{2} \mathrm{As}^{+} \mathrm{GaCl}_{4}^{-}(\mathbf{3 ~ b})$ & $\mathrm{X}_{2} \mathrm{Sn}(5 \mathrm{c})[14]$ & \\
$\lambda=346(729)$ & $\lambda=386(1117)$ & \\
$\tilde{\nu}=28653 \mathrm{~cm}^{-1}$ & $\tilde{v}=25906 \mathrm{~cm}^{-1}$ & \\
$\mathrm{X}_{2} \mathrm{As}^{+} \mathrm{InCl}_{4}^{-}(\mathbf{4 b})$ & \\
$\lambda=349(560)$ & & \\
$\tilde{v}=28653 \mathrm{~cm}^{-1}$ & & \\
\hline
\end{tabular}


Besonders auffallend an den Derivaten $2 b-2 d, 3 b-3 d$ und $4 b-4 d$ ist ihre Farbigkeit, während die Phosphorderivate $\mathbf{2 a}$ und $\mathbf{3 a}$ farblos sind. In Tab. 1 ist jeweils das niederenergetischste Maximum im UV-Spektrum angegeben. Betrachtet man zunächst die Reihe $\mathbf{2 b}, \mathbf{3} \mathbf{b}, \mathbf{4 b}$, so fällt auf, daß sich das Maximum ebenso wie die Molextinktion in dieser Reihenfolge kaum ändert. Man kann hieraus den Schluß ziehen, daß der farbgebende Elektronenübergang offenbar im $\mathrm{Me}_{2} \mathrm{Si}\left(\mathrm{N}^{t} \mathrm{Bu}\right)_{2} \mathrm{As}^{+}-$Kation (zur Struktur der Homologen siehe weiter hinten) erfolgt und das Anion keine große Rolle spielt. In der Reihe $\mathbf{2 b}, \mathbf{2 c}, \mathbf{2 d}$ wird bei gleichbleibendem Anion das Absorptionsmaximum immer mehr in den sichtbaren Bereich verschoben, so daß $\mathbf{2 b}$ eine hellgelbe, $2 \mathrm{c}$ eine tiefgelbe und $\mathbf{2 d}$ eine rote Farbe besitzt. Diese Farbverschiebung erinnert sehr stark an die Verhältnisse beim Übergang von $\mathrm{Me}_{2} \mathrm{Si}\left(\mathrm{N}^{t} \mathrm{Bu}\right)_{3} \mathrm{Ge}(5 \mathrm{~b}), \mathrm{Me}_{2} \mathrm{Si}\left(\mathrm{N}^{t} \mathrm{Bu}\right)_{2} \mathrm{Sn}$ (5c) schließlich zu $\mathrm{Me}_{2} \mathrm{Si}\left(\mathrm{N}^{t} \mathrm{Bu}\right)_{2} \mathrm{~Pb}(5 \mathrm{j})$ [5]. Auch läßt sich z. B. die Lage der Absorptionsbande und die Größenordnung der Molextinktion beim Stannylenderivat [14] sehr gut mit derjenigen der Antimonverbindung 2e vergleichen (s. Tab. 1). Man kann hieraus mit gewisser Einschränkung ableiten, daß die elektronischen Verhältnisse in den Kationen der Verbindungen $\mathbf{2 b}-\mathbf{d}, \mathbf{3} \mathbf{b}-\mathbf{d}$ und $\mathbf{4} \mathbf{b}-\mathbf{d}$ ähnlich denen in den ungeladenen Verbindungen $\mathbf{5 b}-\mathbf{d}$ sein sollten, was wegen der vorliegenden Isosterie auch nicht verwundert.

Tabelle 2 Daten zu den Kristallen und Strukturbestimmungen von $\mathrm{Me}_{2} \mathrm{Si}^{\mathrm{t}}\left(\mathrm{Bu}_{2} \mathrm{PAlCl}_{4}(2 \mathrm{a})\right.$, $\mathrm{Me}_{2} \mathrm{Si}\left(\mathrm{N}^{\mathrm{t}} \mathrm{Bu}\right)_{2} \mathrm{SbAlCl}_{4}(\mathbf{2 c})$ und $\mathrm{Me}_{2} \mathrm{Si}\left(\mathrm{N}^{\mathrm{t}} \mathrm{Bu}\right)_{2} \mathrm{BiAlCl}_{4}$ (2d)

$\mathrm{C}_{10} \mathrm{H}_{24} \mathrm{AlCl}_{4} \mathrm{~N}_{2} \mathrm{PSi}(400,2 \mathrm{~g} / \mathrm{mol}$ ) (2a), orthorhombisch, Raumgruppe Pnma, a $=3023,7(9)$, $\mathrm{b}=1001,0(3), \quad \mathrm{c}=1414,6(5) \mathrm{pm}, \quad \mathrm{V}=4282 \cdot 10^{6} \mathrm{pm}^{3}, \quad \mathrm{Z}=8, \quad \mathrm{D}_{\mathrm{x}}=1,24 \mathrm{~g} / \mathrm{cm}^{3}, \quad \mu(\mathrm{MoK} \alpha)=$ $6,6 \mathrm{~cm}^{-1}$, Kristallabmessungen: $0,2 \cdot 0,2 \cdot 0,4 \mathrm{~mm}$.

Vierkreisdiffraktometer (Stoe, Darmstadt), MoK $\alpha$-Strahlung mit Graphitmonochromator, $\omega / 2 \Theta-$ Scan: 3949 Reflexe, davon 1056 als nichtbeobachtet eingestuft, LP-Korrektur, Reflex/Parameter-Verhältnis $=14,8$; Strukturbestimmung über direkte Methoden (Multan[29]). Gewichtssehema $W=3,23 /\left(\sigma^{2} \mathbf{F}+0,000058 \cdot \mathrm{F}^{2}\right)$. R-Wert: $\Sigma|| \mathrm{F}_{0}|-| \mathrm{F}_{\mathrm{c}}|| / \Sigma\left|\mathrm{F}_{0}\right|=0,069$.

$\mathrm{C}_{10} \mathrm{H}_{21} \mathrm{AlCl}_{4} \mathrm{~N}_{2} \mathrm{SbSi}(491,0 \mathrm{~g} / \mathrm{mol})$ (20), orthorhombisch, Raumgruppe Pbca, $a=2030,8(8)$, $\mathrm{b}=1193,1(4), \quad \mathrm{c}=1777,1(6) \mathrm{pm}, \quad \mathrm{V}=4306 \cdot 10^{6} \mathrm{pm}^{3}, \quad \mathrm{Z}=8, \quad \mathrm{D}_{\mathrm{x}}=1,51 \mathrm{~g} / \mathrm{cm}^{3}, \quad \mu(\mathrm{MoK} \alpha)=$ $18,85 \mathrm{~cm}^{-1}$, Kristallabmessungen: $0,1 \cdot 0,1 \cdot 1 \mathrm{~mm}^{3}$.

Vierkreisdiffraktometer (Stoe, Darmstadt), MoK $\alpha$-Strahlung mit Graphitmonochromator, $\omega / 2 \Theta_{-}$Scan im Meßbereich $4<2 \Theta<45^{\circ} ; 1593$ Reflexe, davon 78 als ,nichtbeobachtet" eingestuft $\left(\mathbf{F}_{0}<3 \sigma \mathbf{F}_{0}\right)$, LP-Korrektur, Reflex/Parameter-Verhältnis $=8,8$; Strukturbestimmung über Sehweratommethoden. R-Wert: $\Sigma|| \mathbf{F}_{0}|-| \mathbf{F}_{\mathrm{c}}|/ \Sigma| \mathbf{F}_{0} \mid=0,093$.

$\mathrm{C}_{10} \mathrm{H}_{24} \mathrm{AlBiCl}_{4} \mathrm{~N}_{2} \mathrm{Si}(578,2 \mathrm{~g} / \mathrm{mol}$ (2d), orthorhombisch, Raumgruppe Pbca, a $=2025,9(8)$, $\mathrm{b}=1198,0(4), \mathrm{c}=1761,3(6) \mathrm{pm}, \mathrm{V}=4275 \cdot 10^{6} \mathrm{pm}^{3}, \mathrm{Z}=8, \mathrm{D}_{\mathrm{x}}=1,79 \mathrm{~g} / \mathrm{cm}^{3}, \mu($ MoK $\alpha)=$ $87,63 \mathrm{~cm}^{-1}$, Kristallabmessungen: $0,4 \cdot 0,3 \cdot 0,1 \mathrm{~mm}$,

Vierkreisdiffraktometer (Siemens, AED 2), MoK $x$-Strahlung mit Graphitmonochromator, $\omega$-Scan im Meßbereich $3<2 \Theta<40^{\circ} ; 1953$ Reflexe, Reflex/Parameter-Verhältnis = 9,8; empirische Absorptionskorrektur über PSI-Scans; LP-Korrektur; Strukturbestimmung über Schweratommethoden; Gewichtsschema: $W=1,003 /\left(\sigma^{2} \mathrm{~F}+0,0001 \cdot \mathbf{F}^{2}\right)$. R-Wert: $\Sigma|| \mathrm{F}_{\mathrm{o}}|-| \mathrm{F}_{\mathrm{e}}|| \Sigma\left|\mathbf{F}_{\mathrm{o}}\right|=0,044$. 
Alle Derivate 2, 3 und 4 liefern richtige Analysenwerte, einige wenige lassen sich sogar im Massenspektrometer in der Gasphase anhand des Molpeaks nachweisen (s. exp. Teil). Letzterer Befund zeigt nochmals, daß der Assoziationsgrad zwischen dem formalen Kation und Anion sehr groß ist und sich die Struktur im Festkörper (siehe nächstes Kapitel) durchaus von derjenigen in Lösung bzw. Gasphase unterscheiden könnte. Die relativ hohen Intensitäten für die Kationen $\mathrm{Me}_{2} \mathrm{Si}\left(\mathrm{N}^{\mathrm{t}} \mathrm{Bu}\right)_{2} \mathrm{El}^{+}$im Massenspektrum belegen jedoch andererseits auch deren besondere Stabilität.

\section{Strukturbestimmungen an $\mathrm{Me}_{2} \mathrm{Si}\left(\mathrm{N}^{\mathrm{t}} \mathrm{Bu}\right)_{2} \mathrm{El}^{+} \mathrm{AlCl}_{4}{ }^{-}(\mathrm{El}=\mathrm{P}(2 \mathrm{a}), \mathrm{Sb}(2 \mathrm{e})$ und $\mathrm{Bi}(2 \mathrm{~d}))$}

Einkristalle von 2a, 2e und $2 \mathbf{d}$ wurden beim Einengen von verdünnten Benzollösungen erhalten ( $2 \mathrm{~b}$ bildet stark verwachsene, sehr kleine Kristalle und konnte daher nicht untersucht werden). Aus Filmaufnahmen wurden die Kristallsysteme und Raumgruppen abgeleitet, während die Reflexintensitäten an automatischen Einkristalldiffraktometern ermittelt wurden (20 Reflexe im Bereich $20^{\circ}<2 \Theta<30^{\circ}$ dienten zur Bestimmung der Zellkonstanten). Alle Details zur Strukturbestimmung und zu den Kristallen sind in der Tab. 2 zusammengestellt. Die Ergebnisse der Strukturbestimmungen sind in den Tab. 3-5 enthalten. $\mathrm{F}_{0} / \mathrm{F}_{\mathrm{c}}$-Listen sowie die anisotropen Temperaturfaktoren sind in den Originaldissertationen aufgenommen (2a [15], 2e und 2d [16]; Strukturfaktoren aus dem SHELXSystem [28]).

Tabelle 3 Die Ortskoordinaten und der Parameter des Debye-Waller-Faktors für die Atome der asymmetrisehen Einheit von $\mathrm{Me}_{2} \mathrm{Si}(\mathrm{NtBu})_{2} \mathrm{PAlCl}_{4}(\mathbf{2})$. Die Standardabweichungen in Klammern beziehen, sich (wie in den Tab. 4 und 5) auf die letzten Stellen der Zahlenwerte. Die angegebenen $\mathbf{B}$-Werte des Temperaturfaktors $\exp \left(-\mathbf{B} \cdot \sin ^{2} \theta / \lambda^{2}\right)$ worden nach Hamiton [27] aus den $u_{i j}$-Werten der Atome [15] berechnet

\begin{tabular}{|c|c|c|c|c|}
\hline Atom & $\mathbf{x} / \mathbf{a}$ & $y / b$ & $\mathrm{z} / \mathrm{c}$ & B $\left[10^{4} \mathrm{pm}^{2}\right]$ \\
\hline $\mathbf{P}(\mathbf{1})$ & $0,9382(\mathbf{1})$ & 0,2500 & $0,7143(2)$ & $3,6(2)$ \\
\hline $\operatorname{Si}(1)$ & $0,9988(\mathbf{1})$ & 0,2500 & $0,8374(2)$ & $3,6(2)$ \\
\hline$C(1)$ & $0,0244(3)$ & $0,4031(8)$ & $0,8862(5)$ & $6,5(6)$ \\
\hline$N(1)$ & $0,9921(2)$ & 0,2500 & $0,7111(5)$ & $3,7(5)$ \\
\hline $\mathrm{N}(2)$ & $0,9398(2)$ & 0,2500 & $0,8314(5)$ & $3,7(5)$ \\
\hline$C(01)$ & $0,0218(3)$ & 0,2500 & $0,6248(8)$ & $5,1(8)$ \\
\hline $\mathrm{C}(02)$ & $0,0509(3)$ & $0,3746(8)$ & $0,6285(6)$ & $7,5(8)$ \\
\hline$C(03)$ & $0,9928(4)$ & 0,2500 & $0,5365(7)$ & $7(1)$ \\
\hline$C(11)$ & $0,9000(4)$ & 0,2500 & $0,8971(8)$ & $4,6(8)$ \\
\hline$C(12)$ & $0,9013(4)$ & $0,374(1)$ & $0,954(1)$ & $13(1)$ \\
\hline$C(13)$ & $0,8584(4)$ & 0,2500 & $0,8398(8)$ & $8(2)$ \\
\hline $\mathrm{P}(2)$ & $0,8175(1)$ & 0,2500 & $0,2274(2)$ & $4,0(2)$ \\
\hline $\mathrm{Si}(2)$ & $0,7561(1)$ & 0,2500 & $0,3499(2)$ & $3,6(2)$ \\
\hline $\mathrm{C}(2)$ & $0,7320(2)$ & $0,4043(8)$ & $0,3991(6)$ & $6,1(6)$ \\
\hline $\mathrm{N}(21)$ & $0,8152(2)$ & 0,2500 & $0,3427(5)$ & $3,8(5)$ \\
\hline$N(22)$ & $0,7629(3)$ & 0,2500 & $0,2235(5)$ & $3,6(6)$ \\
\hline $\mathrm{C}(21)$ & $0,8548(4)$ & 0,2500 & $0,4108(8)$ & $4,6(8)$ \\
\hline$C(22)$ & $0,8982(4)$ & 0,2500 & $0,3490(9)$ & $8(1)$ \\
\hline$C(23)$ & $0,8507(3)$ & $0,1251(9)$ & $0,4710(6)$ & $7,9(8)$ \\
\hline$C(31)$ & $0,7351(4)$ & 0,2500 & $0,1362(8)$ & $4,6(8)$ \\
\hline $\mathrm{C}(32)$ & $0,7662(4)$ & 0,2500 & $0,0499(8)$ & $8(1)$ \\
\hline$C(B 3)$ & $0,7058(3)$ & $0,3735(8)$ & $0,1407(6)$ & $8,2(8)$ \\
\hline Al(1) & $0,0637(\mathbf{1})$ & 0,2500 & $0,2485(2)$ & $4,5(2)$ \\
\hline $\mathrm{Cl}(01)$ & $0,0752(1)$ & $0,0753(2)$ & $0,3324(2)$ & $7,4(2)$ \\
\hline $\mathrm{Cl}(02)$ & $0,1067(1)$ & 0,2500 & $0,1296(3)$ & $9,9(4)$ \\
\hline $\mathrm{Cl}(03)$ & $-0,0029(1)$ & 0,2500 & $0,2030(4)$ & $11,8(5)$ \\
\hline $\mathrm{Al}(2)$ & $0,6876(1)$ & 0,2500 & $0,7126(2)$ & $4,5(2)$ \\
\hline $\mathrm{Cl}(11)$ & $0,6576(1)$ & $0,4248(3)$ & $0,6564(2)$ & $9,0(3)$ \\
\hline $\mathrm{Cl}(12)$ & $0,6771(1)$ & 0,2500 & $0,8626(2)$ & $9,6(4)$ \\
\hline $\mathrm{Cl}(13)$ & $0,7566(1)$ & 0,2500 & $0,6799(3)$ & $9,7(5)$ \\
\hline
\end{tabular}


Tabelle 4 Die Ortskoordinaten und der Parameter des Debye-Waller-Faktors für die Atome der asymmetrischen Finheit von $\mathrm{Me}_{2} \mathrm{Si}(\mathrm{N}: \mathrm{Bu})_{2} \mathrm{SbAlCl}_{4}(2 \mathrm{e})$

Die angegebenen $\mathbf{B}$-Werte des Temperaturfaktors $\exp \left(-\mathbf{B} \cdot \sin ^{2} \theta / \lambda^{2}\right)$ wurden nach HAmitox $[27]$ aus den $u_{i j}-$ Werten der Atome [16] berechnet

\begin{tabular}{|c|c|c|c|c|}
\hline Atom & $\mathrm{x}$ & $\mathrm{y}$ & $\mathrm{z}$ & $\mathrm{B}\left[10^{4} \mathrm{pm}^{2}\right]$ \\
\hline sb & $0,1615(1)$ & $0,1760(2)$ & $0,1486(1)$ & $4,44(7)$ \\
\hline $\mathrm{si}$ & $0,0785(3)$ & $0,2662(8)$ & $0,2613(4)$ & $4,9(4)$ \\
\hline $\mathrm{N}(1)$ & $0,1574(9)$ & $0,220(2)$ & $0,257(1)$ & $6(1)$ \\
\hline$X(2)$ & $0,0722(9)$ & $0,242(2)$ & $0,163(1)$ & $4(1)$ \\
\hline Al & $0,3276(4)$ & $0,3650(9)$ & $0,0775(5)$ & $5,3(4)$ \\
\hline $\operatorname{cl}(1)$ & $0,3433(4)$ & $0,1957(8)$ & $0,0648(5)$ & $7,4(5)$ \\
\hline $\mathrm{Cl}(2)$ & $0 ; 3484(4)$ & $0,4607(8)$ & $-0,0199(4)$ & $6.9(4)$ \\
\hline $\operatorname{dil}(3)$ & $0,3865(4)$ & $0,4338(9)$ & $0,1705(4)$ & $7,3(5)$ \\
\hline $\mathrm{Cl}(4)$ & $0,2265(3)$ & $0,3969(8)$ & $0,1090(5)$ & $6,8(4)$ \\
\hline c(1) & $0,209(1)$ & $0,176(4)$ & $0,317(1)$ & $8(2)$ \\
\hline$c(11)$ & $0,271(1)$ & $0,134(4)$ & $0,279(1)$ & $10(4)$ \\
\hline$(\times 12)$ & $0,180(2)$ & $0,076(3)$ & $0,362(2)$ & $11(3)$ \\
\hline$c(13)$ & $0,214(2)$ & $0,279(3)$ & $0,373(2)$ & $13(3)$ \\
\hline$c(2)$ & $0,020(1)$ & $0,275(3)$ & $0,102(1)$ & $6(2)$ \\
\hline$(x(21)$ & $0,027(1)$ & $0,186(3)$ & $0,037(1)$ & $8(2)$ \\
\hline$(\times(22)$ & $-0,045(1)$ & $0,272(4)$ & $0,141(2)$ & $9(2)$ \\
\hline$C(23)$ & $0,036(1)$ & $0,383(4)$ & $0,072(2)$ & $12(3)$ \\
\hline$C(3)$ & $0,019(1)$ & $0,183(3)$ & $0,318(1)$ & $6(2)$ \\
\hline (14) & $0,078(2)$ & $0,414(4)$ & $0,286(2)$ & $10(3)$ \\
\hline
\end{tabular}

Tabelle 5 Die Ortskoordinaten und der Parameter des Debye-Waller-Faktors für die Atome der asymmetrischen Linheit von $\mathrm{Me}_{2} \mathrm{Si}\left(\mathrm{Nt}_{\mathrm{Bu}}\right)_{2} \mathrm{BiAlCl}_{4}(2 \mathrm{~d})$. Die angegebenen B-Werte (les Tenperaturfaktors $\exp \left(-\mathrm{B} \cdot \operatorname{sij} \iota^{2} \theta / \lambda^{2}\right)$ wurdelt nach Hamlitox [27] aus den $u_{i j}$-Werten der Atome [16] berechnet

\begin{tabular}{|c|c|c|c|c|}
\hline Atom & $\mathrm{x}$ & $\mathrm{y}$ & z & $\mathbf{B}\left[10^{4} \mathrm{pm}^{2}\right]$ \\
\hline $\mathrm{Bi}$ & $0,1624(1)$ & $0,1717(1)$ & $0,1450(1)$ & $3.64(3)$ \\
\hline $\mathrm{Si}$ & $0,0782(2)$ & $0,2663(3)$ & $0,2603(2)$ & $3,9(2)$ \\
\hline$N(1)$ & $0,1557(4)$ & $0,2080(9)$ & $0,2602(6)$ & $3,9(5)$ \\
\hline$N(2)$ & $0,0700(4)$ & $0,2440(8)$ & $0.1630(6)$ & $3,3(6)$ \\
\hline$A 1$ & 0,3264 & $0,3643(3)$ & $0,0757(3)$ & $4,2(2)$ \\
\hline$(l(1)$ & $0,3393(2)$ & $0,1916(3)$ & $0,0624(3)$ & $7,0(2)$ \\
\hline$(11(2)$ & $0,3 \mathbf{4} 90(2)$ & $0,4612(4)$ & $-0,0198(2)$ & $6,4(2)$ \\
\hline$(1)(3)$ & $0,3847(2)$ & $0,4276(3)$ & $0,1681(2)$ & $6,5(2)$ \\
\hline$(1)$ & $0,2255(2)$ & $0,3979(3)$ & $0,1065(3)$ & $6,3(2)$ \\
\hline (1) & $0,2053(7)$ & $0,176(1)$ & $0,3193(9)$ & $5,4(8)$ \\
\hline$C^{\prime}(11)$ & $0,286(1)$ & $0,147(2)$ & $0,285(1)$ & $10,5(6)$ \\
\hline$(12)$ & $0,189(1)$ & $0,075(2)$ & $0,362(1)$ & $10,1(6)$ \\
\hline$(13)$ & $0,209(1)$ & $0,268(2)$ & $0,374(1)$ & $11,5(6)$ \\
\hline$I^{\prime}(2)$ & $0,0207(7)$ & $0,272(1)$ & $0,1052(9)$ & $4,7(7)$ \\
\hline$c^{\prime}(21)$ & $0,0225(9)$ & $0,186(1)$ & $0,041(1)$ & $7,7(5)$ \\
\hline$(122)$ & $-0,0508(9)$ & $0,270(1)$ & $0,144(1)$ & $8,8(5)$ \\
\hline$C^{\prime}(23)$ & $0,034(1)$ & $0,386(1)$ & $0,072(1)$ & $10,2(6)$ \\
\hline (:(3) & $0,0180(7)$ & $0,185(1)$ & $0,3177(9)$ & $5,9(9)$ \\
\hline$(1)$ & $0,0757(6)$ & $0,414(1)$ & $0,288(1)$ & $5,6(7)$ \\
\hline
\end{tabular}

\section{Diskussion der Strukturen}

Wiihrend die Verbindungen $\mathbf{2}$ end $\mathbf{2 d}$ isotyp kristallisieren und isostrukturell sind, besitzt $\mathbf{2 a}$ eine gänzlich andere Kristallstruktur. Im Falle von $\mathbf{2 e}$ und $\mathbf{2 d}$ bestehen intermolekulare Kontakte zwischen den Kationen und Anionen über $\mathrm{Cl} \ldots \mathrm{El}$ ( $\mathrm{El}=\mathrm{Sb}, \mathrm{Bi}$ ) Wechselwirkungen (s. weiter unten), wohingegen bei $\mathbf{2 a}$ 


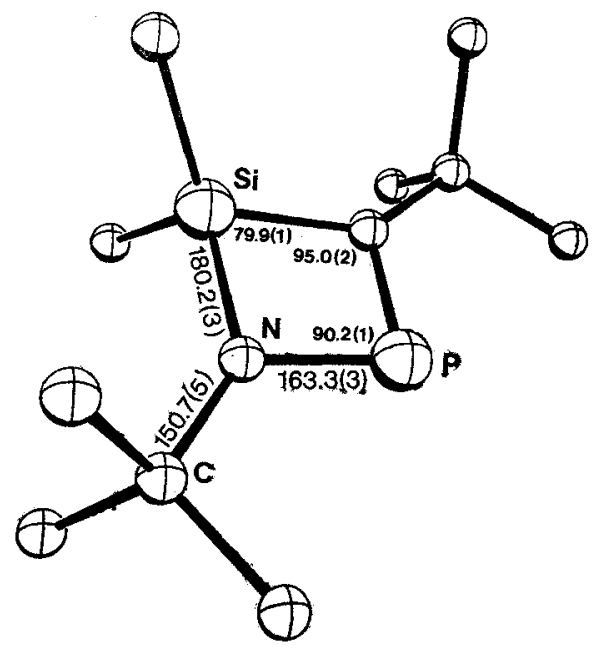

Abb. 1 Ein aus den beiden symmetrieunabhängigen Kationen in $\mathbf{2 a}, \mathbf{M e}_{2} \mathrm{Si}\left(\mathrm{N}^{\mathrm{t}} \mathrm{Bu}\right)_{2} \mathrm{P}^{+}$, , ,gemitteltes ${ }^{\mathrm{i}}$ 。 Molekül mit Bindungslängen (pm) und Winkel $\left({ }^{\circ}\right)$ für die wichtigsten Atome.

Kation und Anion nicht überbrückt sind (der kürzeste P...Cl Kontakt beträgt $336 \mathrm{pm}$ und befindet sich damit außerhalb des Bereichs einer bindenden Wechselwirkung [17]). In Abb. 1 ist ein Kation $\mathrm{Me}_{2} \mathrm{Si}\left(\mathrm{N}^{t} \mathrm{Bu}\right)_{2} \mathrm{P}^{+}$dargestellt, das sich aus der Mittelung der beiden symmetrieunabhängigen Einheiten in der Elementarzelle ergibt. Während die beiden angesprochenen Einheiten jeweils auf der kristallographischen Spiegelebene liegen, besitzt das ,gemittelte "Molekül $\mathrm{C}_{2 \mathrm{v}}(\mathrm{mm} 2)$-Symmetrie (wie ein Vergleich der Bindungslängen und -winkel in Tab. 6 zeigt, ist die Abweichung von der $\mathrm{C}_{2 \mathrm{v}}$-Symmetrie bei beiden Kationen nur unbedeutend). Auch die $\mathrm{AlCl}_{4}$-Anionen nehmen die spezielle Lage der Spiegelebene ein; Abstände und Winkel belegen jedoch, daß auch in diesem Fall die höhere Punktsymmetrie $\operatorname{Td}(\overline{4} 3 \mathrm{~m})$ nahezu erfïllt ist (Tab. 6). Die Sehwerpunkte aller Anionen und Kationen befinden sich somit auf den Spiegelebenen der Elementarzelle $(y=1 / 4$ und 3/4) und sind so zueinander ausgerichtet, daß die tert-Butylgruppen einen möglichst großen Abstand voneinander einnehmen.

Im Gegensatz zu den weiter unten zu diskutierenden Strukturen sind die Anionen and Kationen in $\mathbf{2 a}$ weitgehend isoliert voneinander, wobei innerhalb der Schichten (Spiegelebenen) van der Waals-Kontakte vorherrschen und in Richtung der b-Achse nur schwache Wechselwirkungen über Chlorbrücken auftreten. Im Kation fallen folgende Besonderheiten auf: das Phosphoratom ist nur zweifach koordiniert, die Stickstoffatome befinden sich im Zentrum einer trigonal planaren Anordnung und der $\mathrm{N}-\mathrm{Si}-\mathrm{N}$-Winkel ist spitzer als der $\mathrm{N}-\mathrm{P}-\mathrm{N}$ Winkel (s. Tab. 6). Der gemittelte $\mathrm{P}-\mathrm{N}$-Abstand von $163,3 \mathrm{pm}$ ist um etwa $10 \mathrm{pm}$ kürzer als ein $\mathrm{P}-\mathrm{N}$-Einfachbindungsabstand $[17,18]$ und gut vergleichbar mit offenkettigen Bis(amino)phosphoniumkationen [8]. Die starke Elektronenabgabe vom Stickstoff zum Phosphor im Sinne einer intramolekularen $\pi$-Bindung 
Tabelle 6 Die wichtigsten Abstände (pm) und Winkel $\left(^{\circ}\right)$ in $\mathrm{Me}_{2} \mathrm{Si}\left(\mathrm{N}^{\mathrm{t}} \mathrm{Bu}\right)_{2} \mathrm{PAlCl}_{4}(\mathbf{2 a})$ Bei den Standardabweichungen sind die Ungenauigkeiten in den Ortskoordinaten berücksichtigt

\begin{tabular}{|c|c|c|c|}
\hline$P(1)-N(1)$ & $161,9(7)$ & $P(2)-N(21)$ & $163,5(8)$ \\
\hline $\mathbf{P}(1)-\mathbf{N}(2)$ & $163,8(8)$ & $\mathbf{P}(2)-\mathbf{N}(22)$ & $163,9(8)$ \\
\hline $\mathrm{Si}(1)-\mathrm{N}(1)$ & $178,9(8)$ & $\mathrm{Si}(2)-\mathrm{N}(21)$ & $180,3(8)$ \\
\hline $\mathbf{S i}(1)-\mathbf{N}(2)$ & $180,6(8)$ & $\mathrm{Si}(2)-\mathbf{N}(22)$ & $180,8(8)$ \\
\hline $\mathrm{Si}(1)-\mathrm{C}(1)$ & $185,2(8)$ & $\mathrm{Si}(2)-\mathrm{C}(2)$ & $185,3(8)$ \\
\hline $\mathrm{N}(1)-\mathrm{C}(01)$ & $152(1)$ & $\mathrm{N}(21)-\mathrm{C}(21)$ & $151(1)$ \\
\hline $\mathrm{N}(2)-\mathrm{C}(11)$ & $149(1)$ & $\mathrm{N}(22)-\mathrm{C}(31)$ & $151(1)$ \\
\hline $\mathrm{C}(01)-\mathrm{C}(02)$ & $154(1)$ & $\mathrm{C}(21)-\mathrm{C}(22)$ & $1 \breve{6} 6(2)$ \\
\hline $\mathrm{C}(01)-\mathrm{C}(03)$ & $155(1)$ & $\mathrm{C}(21)-\mathrm{C}(23)$ & $152(1)$ \\
\hline $\mathrm{C}(11)-\mathrm{C}(12)$ & $150(1)$ & $\mathrm{C}(31)-\mathrm{C}(32)$ & $157(2)$ \\
\hline$C(11)-C(13)$ & $155(1)$ & $\mathrm{C}(31)-\mathrm{C}(33)$ & $154(1)$ \\
\hline $\mathrm{Al}(1)-\mathrm{Cl}(01)$ & $213,6(3)$ & $\mathrm{Al}(2)-\mathrm{Cl}(11)$ & $212,5(3)$ \\
\hline $\mathrm{Al}(1)-\mathrm{Cl}(02)$ & $211,4(5)$ & $\mathrm{Al}(2)-\mathrm{Cl}(12)$ & $212,5(5)$ \\
\hline $\mathrm{Al}(1)-\mathrm{Cl}(03)$ & $210,8(5)$ & $\mathrm{Al}(2)-\mathrm{Cl}(13)$ & $212,9(5)$ \\
\hline $\mathbf{N}(2)-\mathbf{P}(1)-\mathbf{N}(1)$ & $90,0(4)$ & $\mathbf{N}(22)-\mathrm{P}(2)-\mathrm{N}(21)$ & $90,4(4)$ \\
\hline $\mathrm{N}(2)-\mathrm{Si}(1)-\mathrm{N}(1)$ & $79,7(3)$ & $N(22)-S i(2)-N(21)$ & $80,1(4)$ \\
\hline$N(1)-S i(1)-C(1)$ & $115,4(3)$ & $\mathrm{N}(21)-\mathrm{Si}(2)-\mathrm{C}(2)$ & $114,5(3)$ \\
\hline$N(2)-S i(1)-C(1)$ & $115,0(3)$ & $\mathrm{N}(22)-\mathrm{Si}(2)-\mathrm{C}(2)$ & $114,5(3)$ \\
\hline$C(1)-S i(1)-C\left(1^{\prime}\right)$ & $112,7(5)$ & $\mathrm{C}(2)-\mathrm{Si}(2)-\mathrm{C}\left(2^{\prime}\right)$ & $114,2(5)$ \\
\hline $\operatorname{Si}(1)-N(1)-\mathbf{P}(1)$ & $95,8(4)$ & $\mathrm{Si}(2)-\mathrm{N}(21)-\mathrm{P}(2)$ & $94,9(4)$ \\
\hline $\mathrm{C}(01)-\mathbf{N}(1)-\mathbf{P}(1)$ & $127,6(6)$ & $\mathrm{C}(21)-\mathrm{N}(21)-\mathbf{P}(2)$ & $126,3(6)$ \\
\hline $\mathrm{C}(01-\mathrm{N}(1)-\mathrm{Si}(1)$ & $136,6(6)$ & $\mathrm{C}(21)-\mathrm{N}(21)-\mathrm{Si}(2)$ & $138,8(6)$ \\
\hline $\mathrm{Si}(1)-N(2)-P(1)$ & $94,5(4)$ & $\mathrm{Si}(2)-\mathrm{N}(22)-\mathrm{P}(2)$ & $94,6(4)$ \\
\hline $\mathrm{C}(11)-\mathbf{N}(2)-\mathbf{P}(1)$ & $127,5(7)$ & $\mathrm{C}(31)-\mathrm{N}(22)-\mathrm{P}(2)$ & $127,2(7)$ \\
\hline $\mathrm{C}(11)-\mathrm{N}(2)-\mathrm{Si}(1)$ & $138,0(6)$ & $\mathrm{C}(31)-\mathrm{N}(22)-\mathrm{Si}(2)$ & $138,3(7)$ \\
\hline $\mathrm{Cl}(02)-\mathrm{Al}(1)-\mathrm{Cl}(01)$ & $109,8(1)$ & $\mathrm{Cl}(12)-\mathrm{Al}(2)-\mathrm{Cl}(11)$ & $108,0(1)$ \\
\hline $\mathrm{Cl}(03)-\mathrm{Al}(1)-\mathrm{Cl}(01)$ & $109,2(1)$ & $\mathrm{Cl}(13)-\mathrm{Al}(2)-\mathrm{Cl}(11)$ & $109,5(1)$ \\
\hline $\mathrm{Cl}(01)-\mathrm{Al}(1)-\mathrm{Cl}\left(01^{\prime}\right)$ & $109,2(2)$ & $\mathrm{Cl}(11)-\mathrm{Al}(2)-\mathrm{Cl}\left(11^{\prime}\right)$ & $110,9(2)$ \\
\hline
\end{tabular}

läßt sich auch aus der relativ langen $\mathrm{Si}-\mathrm{N}$-Bindung ablesen, die mit $180 \mathrm{pm}$ außergewöhnlich aufgeweitet erscheint [19]. Die Elektronenpaare an den Stickstoffatomen werden demnach vom Phosphoratom stärker beansprucht als vom Siliciumatom. Vereinfachend kann man die Bindungsverhältnisse mit den Resonanzformeln $6 \mathrm{a}$ und $6 \mathrm{~b}$ beschreiben.

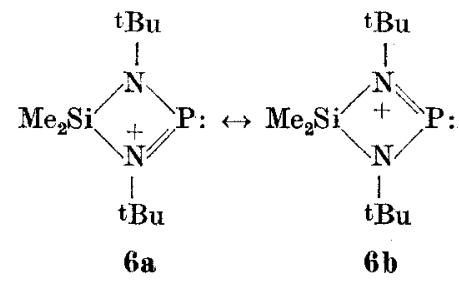

Nach $6 \mathrm{a}$ und $6 \mathrm{~b}$ sollten den $\mathrm{P}-\mathrm{N}$-Bindungen eine Bindungsordnung von eineinhalb zukommen. Verglichen mit Abständen in Organylphosphiniminen sind sie in $2 \mathrm{a}$ um etwa 7 pm länger [20], wobei diese Verbindungen jedoch Phos- 
phor der $\mathrm{KZ}=4$ und der Oxidationszahl +5 enthalten. Bildet man das arithmetische Mittel aus $\mathrm{N}=\mathrm{N}$ - und $\mathrm{P}=\mathrm{P}$-Bindungslängen [21], so findet man einen Erwartungswert für eine $\mathrm{P}=\mathrm{N}$-Bindung mit einem Phosphoratom der $\mathrm{KZ}=2$ und der Oxidationszahl +3 von etwa $162 \mathrm{pm}$.

Die isotypen Kristallstrukturen der Derivate $2 \mathrm{e}$ und $\mathbf{2 d}$ unterscheiden sich von $2 \mathrm{a}$ insbesondere in andersartiger Wechselwirkung zwischen den Kationen $\mathrm{Me}_{2} \mathrm{Si}\left(\mathrm{N}^{t} \mathrm{Bu}\right)_{2} \mathrm{El}^{+}$und den $\mathrm{AlCl}_{4}$-Anionen. Eine $\mathrm{AlCl}_{4}$-Einheit wirkt über die Chloratome $\mathrm{Cl}(3)$ und $\mathrm{Cl}(4)$ als Brücke zwischen zwei $\mathrm{Me}_{2} \mathrm{Si}\left(\mathrm{N}^{t} \mathrm{Bu}\right)_{2}$ El-Kationen, welche ihrerseits zwei $\mathrm{AlCl}_{4}$-Anionen überbrücken. Das Antimon- und Bismutatom ist demnach immer vierfach koordiniert. In Abb. 2 ist am Beispiel des BiDerivates ein Ausschnitt aus der Struktur wiedergegeben. Es entsteht somit eine unendliche Kette aus Kationen und Anionen, die über Cl-Atome verbunden sind. In den Tab. 7 und 8 sind die wichtigsten Abstände und Winkel in den Molekülen und in der Kristallstruktur zusammengestellt.

Tabelle 7 Abstände (pm) und Winkel $\left(^{\circ}\right)$ in $\mathrm{Me}_{2} \mathrm{Si}\left(\mathrm{N}^{\mathrm{t}} \mathrm{Bu}\right)_{2} \mathrm{SbAlCl}_{4}$ (2c). Standardabweichungen wie in Tab. 6

\begin{tabular}{|c|c|c|c|c|c|c|}
\hline $\mathrm{Sb}-\mathrm{N}(1)$ & $200(2)$ & $\mathrm{N}(1)-\mathrm{Sb}-\mathrm{N}(2)$ & $74,4(7)$ & \multicolumn{2}{|c|}{$\mathrm{Cl}(1)-\mathrm{Al}-\mathrm{Cl}(2)$} & $114,2(5)$ \\
\hline $\mathbf{S b}-\mathbf{N}(2)$ & $199(2)$ & & & \multicolumn{2}{|c|}{$\mathrm{Cl}(1)-\mathrm{Al}-\mathrm{Cl}(3)$} & $111,4(5)$ \\
\hline & & $\mathbf{N}(1)-\mathrm{Si}-\mathbf{N}(2)$ & $88,6(9)$ & \multicolumn{2}{|c|}{$\mathrm{Cl}(1)-\mathrm{Al}-\mathrm{Cl}(4)$} & $110,3(5)$ \\
\hline $\mathrm{Si}-\mathrm{N}(1)$ & $169(2)$ & $\mathrm{N}(1)-\mathrm{Si}-\mathrm{C}(3)$ & $117(1)$ & \multicolumn{2}{|c|}{$\mathrm{Cl}(2)-\mathrm{Al}-\mathrm{Cl}(3)$} & $107,7(5)$ \\
\hline $\mathrm{Si}-\mathbf{N}(2)$ & $177(2)$ & $N(1)-S i-C(4)$ & $109(1)$ & \multicolumn{2}{|c|}{$\mathrm{Cl}(2)-\mathrm{Al}-\mathrm{Cl}(4)$} & $107,8(5)$ \\
\hline $\mathrm{Si}-\mathrm{C}(3)$ & $185(3)$ & $\mathrm{N}(2)-\mathrm{Si}-\mathrm{C}(3)$ & $113(1)$ & \multicolumn{2}{|c|}{$\mathrm{Cl}(3)-\mathrm{Al}-\mathrm{Cl}(4)$} & $104,9(5)$ \\
\hline \multirow[t]{2}{*}{$\mathrm{Si}-\mathrm{C}(4)$} & \multirow[t]{2}{*}{$182(2)$} & $\mathrm{N}(2)-\mathrm{Si}-\mathrm{C}(4)$ & $113(1)$ & & & \\
\hline & & $\mathrm{C}(3)-\mathrm{Si}-\mathrm{C}(4)$ & $112(1)$ & & & \\
\hline $\mathrm{Al}-\mathrm{Cl}(1)$ & $205(1)$ & & & & & \\
\hline $\mathrm{Al}-\mathrm{Cl}(2)$ & $211(1)$ & $\mathrm{Sb}-\mathrm{N}(1)-\mathrm{Si}$ & $99,7(8)$ & & & \\
\hline $\mathrm{Al}-\mathrm{Cl}(3)$ & $219(1)$ & $\mathrm{Sb}-\mathrm{N}(1)-\mathrm{C}(1)$ & $122(2)$ & & & \\
\hline \multirow[t]{2}{*}{$\mathrm{Al}-\mathrm{Cl}(4)$} & \multirow[t]{2}{*}{$216(1)$} & $S i-N(1)-C(1)$ & $135(1)$ & & & \\
\hline & & $\mathrm{Sb}-\mathrm{N}(2)-\mathrm{Si}$ & $97,4(9)$ & & & \\
\hline$N(1)-C(1)$ & $158(3)$ & $\mathrm{Sb}-\mathrm{N}(2)-\mathrm{C}(2)$ & $128(1)$ & & & \\
\hline $\mathrm{N}(2)-\mathrm{C}(2)$ & $156(3)$ & $\mathrm{Si}-\mathbf{N}(2)-\mathrm{C}(2)$ & $134(2)$ & & & \\
\hline \multicolumn{7}{|c|}{ Gemittelte Abstände und Winkel } \\
\hline \multirow{2}{*}{\multicolumn{2}{|c|}{$\begin{array}{l}C(1)-C(11,12,13) \\
C(2)-C(21,22,23)\end{array}$}} & $155(4)$ & \multicolumn{3}{|c|}{$N(1)-C(1)-C(11,12,13)$} & $108(2)$ \\
\hline & & $150(4)$ & $\mathbf{N}(2)$ & $-\mathrm{C}(21,22,23)$ & & $107(2)$ \\
\hline \multicolumn{7}{|c|}{ Intra- und intermolekulare koordinative bzw. „nicht-bindende“ Abstände und Winkel } \\
\hline \multicolumn{2}{|l|}{$\mathrm{Sb} \ldots \mathrm{Cl}(4)$} & $303,1(8)$ & \multirow{2}{*}{\multicolumn{2}{|c|}{$\begin{array}{l}\mathrm{N}(1) \cdots \mathrm{N}(2) \\
\mathrm{Si} \cdots \mathrm{Sb}\end{array}$}} & \multicolumn{2}{|l|}{$242(2)$} \\
\hline \multicolumn{2}{|l|}{$\mathrm{Sb} \cdots \mathrm{Cl}\left(3^{\prime}\right)$} & $307,5(8)$ & & & \multicolumn{2}{|c|}{$283,0(7)$} \\
\hline \multicolumn{2}{|l|}{$\mathrm{Sb} \ldots \mathrm{Cl}(1)$} & $398,8(9)$ & \multicolumn{2}{|c|}{$\mathrm{Si} \cdots \mathrm{Sb}$} & \multirow{2}{*}{\multicolumn{2}{|c|}{$104(1)$}} \\
\hline \multicolumn{2}{|l|}{$\mathrm{Sb} \cdots \mathrm{Cl}\left(2^{\prime}\right)$} & $395,1(9)$ & \multicolumn{2}{|c|}{$\mathrm{C}(3)-\mathrm{Si} \cdots \mathrm{Sb}$} & \multirow{2}{*}{\multicolumn{2}{|c|}{$\begin{array}{l}124(1) \\
122(1)\end{array}$}} \\
\hline $\mathrm{Sb} \ldots \mathrm{Cl}\left(4^{\prime}\right)$ & & $409,5(9)$ & \multicolumn{2}{|c|}{$\mathrm{C}(4)-\mathrm{Si} \ldots \mathrm{Sb}$} & & \\
\hline
\end{tabular}




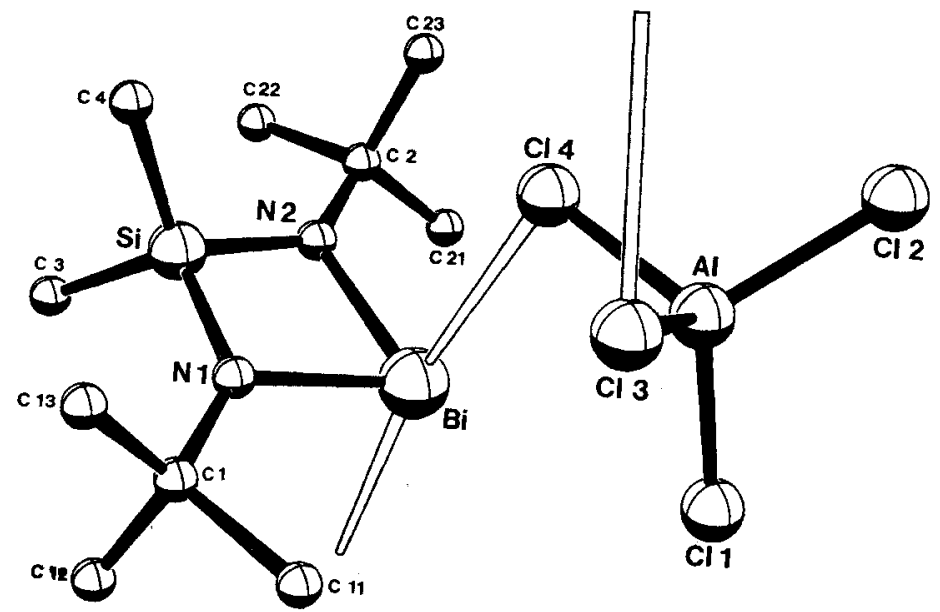

Abb. 2 Aussehnitt aus der Kristallstruktur von $\mathrm{Me}_{2} \mathrm{Si}\left(\mathrm{N}^{\dagger} \mathrm{Bu}\right)_{2} \mathrm{BiAlCl}_{4}$ (2d). Die nicht ausgefüllten Bindungen geben die Wechselwirkungen zwischen $\mathrm{Bi}$ und den Chloratomen $\mathrm{Cl}(4)$ und $\mathrm{Cl}\left(3^{\prime}\right)$ wieder. Die Bindung zum Bi geht von einem nicht gezeichneten $\mathrm{Cl}\left(3^{\prime}\right)$ eines benachbarten $\mathrm{AlCl}_{4}^{-}$ans und $\mathrm{Cl}(3)$ weist auf ein ebenfalls nicht dargestelltes $\mathrm{Bi}^{\prime \prime}$ eines benachbarten $\mathrm{Me}_{2} \mathrm{Si}\left(\mathrm{N}^{t} \mathrm{Bu}\right)_{2} \mathrm{Bi}$-Kations.

Tabelle 8 Abstände $(\mathrm{pm})$ und Winkel $\left({ }^{\circ}\right)$ in $\mathrm{Me}_{2} \mathrm{Si}\left(\mathrm{N}^{\mathrm{t}} \mathrm{Bu}\right)_{2} \mathrm{BiAlCl}_{4}$ (2d). Standardabweichungen wie in Tab. 6

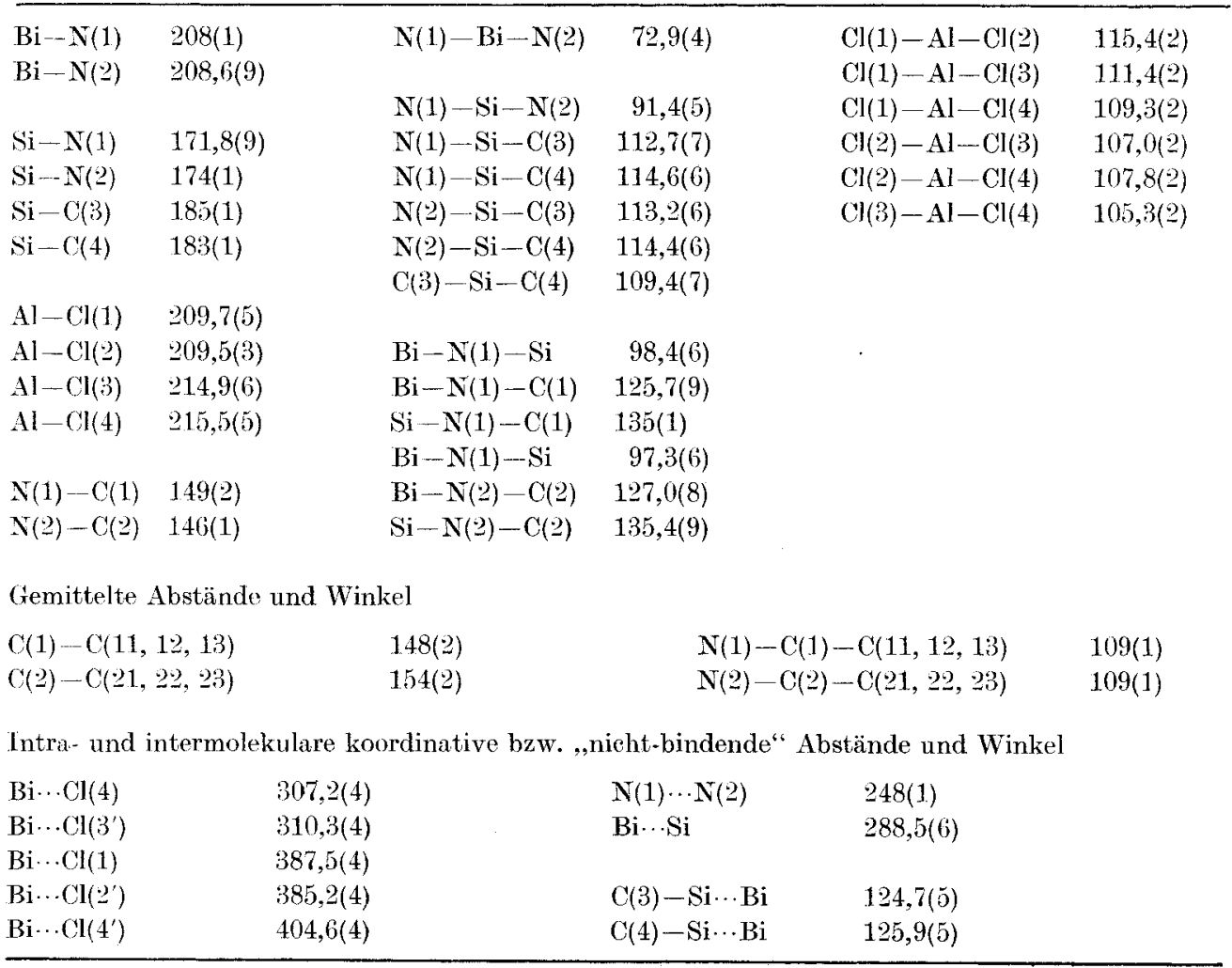


Die Kationen $\mathrm{Me}_{2} \mathrm{Si}\left(\mathrm{N}^{t} \mathrm{Bu}\right)_{2} \mathrm{El}^{+}(\mathrm{El}=\mathrm{Sb}$ und $\mathrm{Bi})$ besitzen wie das entsprechende Kation in $2 \mathbf{a}$ weitgehend $\mathrm{C}_{2 \mathrm{v}}(\mathrm{mm} 2)$-Symmetrie, auch wenn wegen der allgemeinen Lage dieser Einheiten in der Elementarzelle eine relativ große Abweichung hiervon, vor allem beim Sb-Derivat, beobachtet wird (Tab. 7 und 8). Die $\mathrm{AlCl}_{4}$-Anionen in $\mathbf{2 e}$ und $\mathbf{2 d}$ unterscheiden sich hingegen von demjenigen der Struktur 2a deutlich. Die Verzerrung der Tetraedersymmetrie (Td) zu annähernd $\mathrm{C}_{2 \mathrm{v}}$ (die pseudozweizählige Achse ist die Winkelhalbierende des $\mathrm{Cl}(1)-\mathrm{Al}-\mathrm{Cl}(2)-\mathrm{bzw} . \mathrm{Cl}(3)-\mathrm{Al}-\mathrm{Cl}(4)$-Winkels) geht deutlich aus den Abständen und Winkeln der Tabellen 7 und 8 hervor. Von den vier Chlorliganden am Aluminium sind die Atome $\mathrm{Cl}(1)$ und $\mathrm{Cl}(2)$ terminal gebunden, während $\mathrm{Cl}(3)$ und $\mathrm{Cl}(4)$ Brückenfunktionen übernehmen. Dementsprechend findet man in beiden Strukturen für $\mathrm{Al}-\mathrm{Cl}(3)$ bzw. $\mathrm{Al}-\mathrm{Cl}(4)$ um $5-7 \mathrm{pm}$ längere Abstände als für $\mathrm{Al}-\mathrm{Cl}(1,2)$. Die gleichzeitige Annäherung der Liganden $\mathrm{Cl}(3)$ und $\mathrm{Cl}(4)$ an die Sb- bzw. Bi-Atome geht offenbar zu Lasten von Elektronendichte in den $\mathrm{Al}-\mathrm{Cl}(3,4)$-Bindungen und zu Gunsten der Dichte in den $\mathrm{Al}-\mathrm{Cl}(1,2)$-Bindungen. Besonders illustrativ ist ein Vergleich mit den Bindungsverhältnissen in $\mathrm{NaAlCl}_{4}$ $\left(\mathrm{AlCl}_{4}\right.$-Tetraeder mit $\left.\mathrm{Al}-\mathrm{Cl}=213 \mathrm{pm}[22]\right)$ und im $\mathrm{Al}_{2} \mathrm{Cl}_{6}(\mathrm{Al}-\mathrm{Cl}$ (terminal) $=$ $207 \mathrm{pm}, \mathrm{Al}-\mathrm{Cl}($ Brücke $)=225 \mathrm{pm}$ [23]). Die bei $\mathbf{2 c}$ und $\mathbf{2 d}$ beobachteten Verzerrungen liegen demnach zwischen diesen beiden Extremen.

Die als Elektronenakzeptoren auftretenden Atome $\mathrm{Sb}$ und Bi befinden sich in einem Abstand von $303-310 \mathrm{pm}$ von den Chloratomen $\mathrm{Cl}\left(3^{\prime}\right)$ und $\mathrm{Cl}(4)$. Im Vergleich zu den $\mathrm{Sb}-\mathrm{Cl}-\mathrm{bzw}$. Bi-Cl-Abständen in der ersten Koordinationssphäre der kristallinen Elementtrichloride [23] $\left(\mathrm{SbCl}_{3} 234 \mathrm{pm}, \mathrm{BiCl}_{3} 250 \mathrm{pm}\right)$ sind diese Längen deutlich größer, andererseits aber auch kleiner als diejenigen einer zweiten Sphäre $\left(\mathrm{SbCl}_{3} 346 \mathrm{pm}, \mathrm{BiCl}_{3} 321 \mathrm{pm}\right)$. Wir müssen daher davon ausgehen, daß ein erheblicher Anteil der Elektronenarmut an den $\mathrm{Sb}$ - und $\mathrm{Bi}$ Atomen durch die Chlorliganden $\mathrm{Cl}\left(3^{\prime}\right)$ und $\mathrm{Cl}(4)$ kompensiert wird. Parallel hierzu ergeben sich auch kürzere $\mathrm{Si}-\mathrm{N}$-Bindungslängen in den Vierringen der Verbindungen $2 \boldsymbol{e}$ und $2 \mathbf{d}$ als bei der Phosphorverbindung $2 a$ (Tab. 7 und 8). Verglichen mit den Bis(amino)halogeniden $\mathrm{Me}_{2} \mathrm{Si}\left(\mathrm{N}^{t} \mathrm{Bu}\right)_{2} \mathrm{Sb}-\mathrm{Cl}$ und $\mathrm{Me}_{2} \mathrm{Si}\left(\mathrm{N}^{t} \mathrm{Bu}\right)_{2} \mathrm{Bi}-\mathrm{Cl}$ [1] findet man allerdings in den kationischen Spezies weder in den $\mathrm{Si}-\mathrm{N}$-Bindungen noch in der $\mathrm{Sb}-\mathrm{N}$-Bindung eine bemerkenswerte Bindungslängenänderung. Nur der Wert der $\mathrm{Bi}-\mathrm{N}$-Bindung verringert sich geringfügig um $4 \mathrm{pm}$ beim Übergang von $\mathrm{Me}_{2} \mathrm{Si}\left(\mathrm{N}^{t} \mathrm{Bu}\right)_{2} \mathrm{Bi}-\mathrm{Cl}$ zu $\mathrm{Me}_{2} \mathrm{Si}\left(\mathrm{N}^{t} \mathrm{Bu}\right)_{2} \mathrm{BiAlCl}_{4}$. Die Atome $\mathrm{Sb}$ und $\mathrm{Bi}$ in $2 \mathrm{e}$ und $\mathbf{2 d}$ sind im Zentrum trigonal bipyramidaler Koordinationsfiguren, mit den beiden Stickstoffatomen und dem einsamen Elektronenpaar in äquatorialer und den Chlordonatoren in axialer Stellung (der Winkel $\mathrm{Cl}(4)-\mathrm{El}-\mathrm{Cl}\left(3^{\prime}\right)$ beträgt bei beiden Derivaten $171^{\circ}$ ).

Im Unterschied zum Kation $\mathrm{Me}_{2} \mathrm{Si}\left(\mathrm{N}^{t} \mathrm{Bu}\right) \mathrm{P}^{+}$der Verbindung 2a, bei dem die positive Ladung über die beiden Stickstoffatome und das Phosphoratom verteilt jst (Formelbilder 6a und 6b) muß man bei dem entsprechenden Antimon- und Bismut-Derivat aufgrund der gefundenen Struktur eine Konzentration der Ladung am Sb oder Bi annehmen. Die Stickstoffatome beteiligen sich bei $2 \mathrm{e}$ 
und $2 \mathrm{~d}$ demnach relativ wenig an einer Ladungsverteilung und sind weit mehr an das Siliciumatom gebunden als bei $\mathbf{2 a}$.

Versuch einer qualitativen Bindungsbeschreibung in Diaza-sila-element-etidinen auf der Grundlage struktureller Parameter

Die in dieser Arbeit vorgestellten cyclischen Kationen können in eine „Familie" von Verbindungen eingereiht werden, die alle die Bis(tert-butylamino)dimethylsilylgruppe als gemeinsames Bauelement besitzen [24]. Es bietet sich an, die geometrischen Abmessungen dieser Gruppe als "Sonde“ zu benutzen, um indirekt etwas über die Wechselwirkung des Elementes mit den Stickstoffatomen im planaren Diazasilaelementetidin aussagen zu können. Zwei Größen sind hierbei besonders instruktiv: zum einen die $\mathrm{Si}-\mathrm{N}$-Bindungslänge und zum anderen der $\mathrm{N}-\mathrm{Si}-\mathrm{N}$-Winkel. In den Abb. 3 und 4 sind diese Parameter für eine Reihe von Elementen bzw. Elementgruppierungen in zweifacher und dreifacher Koordination (neben den Stickstoffatomen befindet sich noch ein weiterer Ligand am Element) aufgetragen.

Zwei Effekte lassen sich deutlich erkennen: Je größer die Wechselwirkung des Elementes oder der Elementgruppe mit den Stickstoffatomen ist, um so länger fällt die $\mathrm{Si}-\mathrm{N}$-Bindung aus, bzw. um so spitzer ist der $\mathrm{N}-\mathrm{Si}-\mathrm{N}$-Winkel (Konkurrenz zwischen dem Element und Silicium um die Elektronen an den Stickstoffatomen). Im übrigen korrelieren $\mathrm{Si}-\mathrm{N}$-Bindungslänge und $\mathrm{N}-\mathrm{Si}-\mathrm{N}$-Winkel mit-

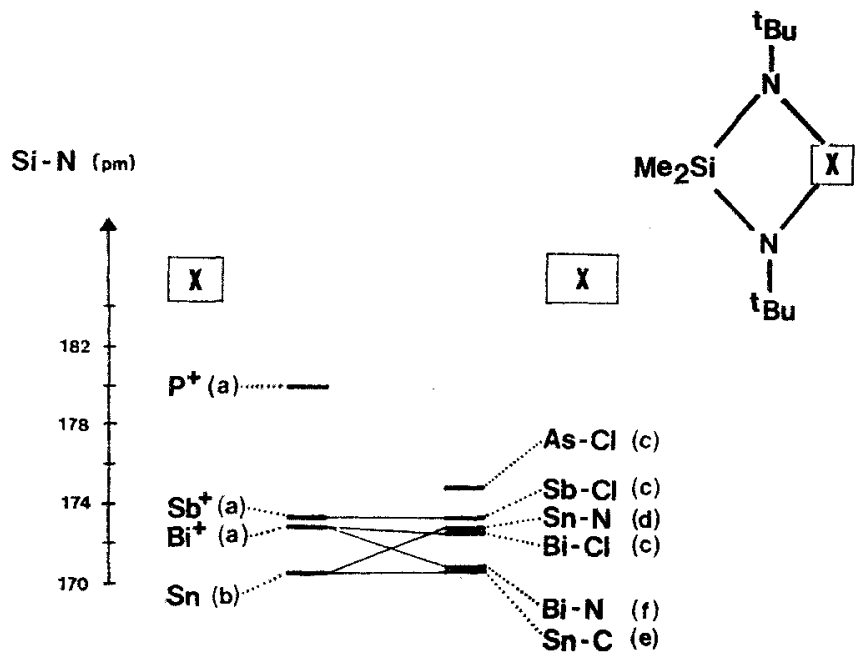

Abb. 3 DieSi-N-Bindungslänge in verschiedenen Vierringen der allgemeinen Formel $\mathrm{Me}_{2} \mathrm{Si}\left(\mathrm{N}^{\mathrm{t}} \mathrm{Bu}\right)_{2} \mathrm{X}$ nach Röntgenstrukturanalysen. a) 2a, 2e, 2d (diese Arbeit), b) 5e [25], c) 1b, 1e, 1d [1],

d) $\mathrm{Me}_{2} \mathrm{Si}\left(\mathrm{N}^{\mathrm{t}} \mathrm{Bu}\right)_{2} \mathrm{Sn} \cdot \mathrm{N}\left({ }^{\mathrm{t}} \mathrm{Bu}\right) \mathrm{H}_{2}[14]$, e) $\mathrm{Me}_{2} \mathrm{Si}\left(\mathrm{N}^{\mathrm{t}} \mathrm{Bu}\right)_{2} \mathrm{Sn} \cdot \mathrm{C}\left(\mathrm{PPh}_{3}\right) \mathrm{H}_{2}[20]$,

f) $\mathrm{Me}_{2} \mathrm{Si}\left(\mathrm{N}^{\mathrm{t}} \mathrm{Bu}\right)_{2} \mathrm{Bi}-\mathrm{N}\left({ }^{\mathrm{t}} \mathrm{Bu}\right) \mathrm{SiMe}_{2}\left({ }^{t} \mathrm{Bu}\right) \mathrm{N}-\mathrm{Bi}\left(\mathrm{N}^{\mathrm{t}} \mathrm{Bu}\right)_{2} \mathrm{SiMe}_{2}$ [1]. Die in der skizze eingeführten Symbole stellen teilweise Kürzel dar (z. B. Sn-N, Sn-C und Bi-N), und sind auch in dieser Weise in den $\mathrm{Abb} .4$ und 5 enthalten. 


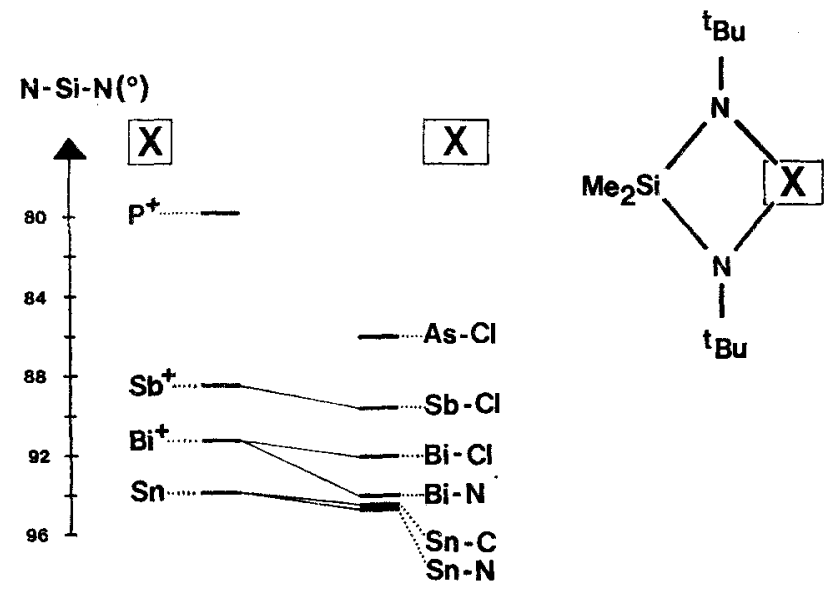

Abb. 4 Der $\mathrm{N}-\mathrm{Si}-\mathrm{N}$-Bindungswinkel in $\mathrm{Me}_{2} \mathrm{Si}\left(\mathrm{N}^{t} \mathrm{Bu}\right)_{2} \mathrm{X}$ nach Röntgenstrukturanalyse. Bezsichnungen wie in Abb. 3. Formal zweifach koordinierte Elemente (links) sind wie in Abb. 3 von formal dreifach koordinierten (rechts) unterschieden.

einander, wie aus $\mathrm{Abb} .5 \mathrm{zu}$ ersehen. Dies ist einfach zu erklären: bei geringerer Wechselwirkung der Stickstoffatome mit den Siliciumatomen ,,wandert" das Siliciumatom aus dem Ring heraus, wobei die Stickstoffatome in relativ gleichem Abstand zueinander verbleiben.

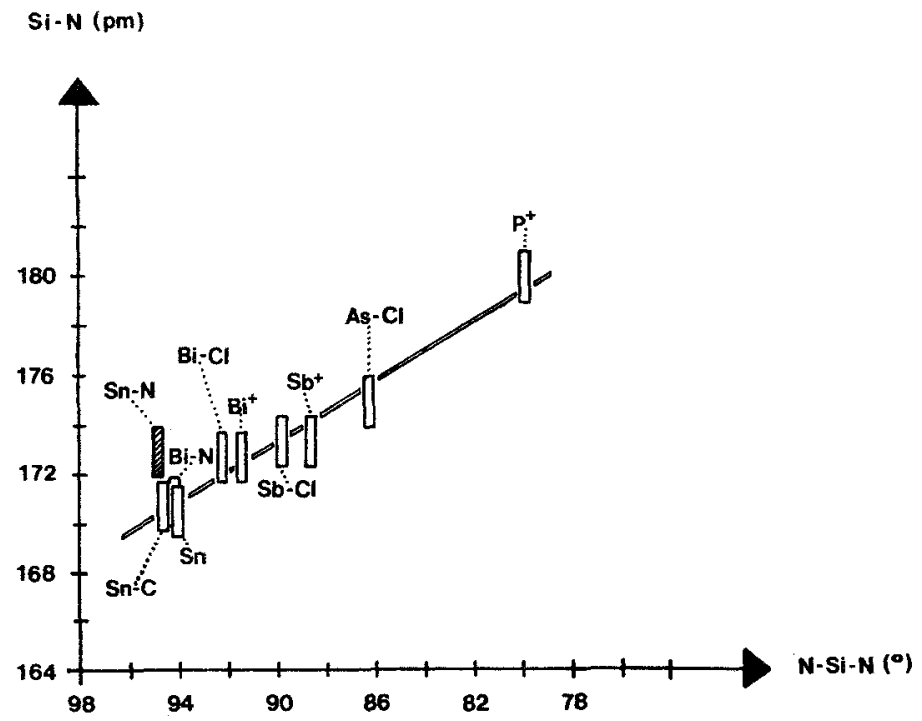

Abb.5 Korrelation zwischen $\mathrm{Si}-\mathrm{N}$-Bindungslänge und $\mathrm{N}-\mathrm{Si}-\mathrm{N}$-Winkel im Vierring $\mathrm{Me}_{2} \mathrm{Si}\left(\mathrm{N}^{\mathrm{t}} \mathrm{Bu}\right)_{2} \mathrm{X}$ (vgl. auch Legenden zu Abb. 3 und 4). Die schraffierte Verbindung $\mathrm{Me}_{2} \mathrm{Si}\left(\mathrm{N}^{t} \mathrm{Bu}\right)_{2} \mathrm{Sn}$. $\mathrm{N}\left({ }^{\mathrm{i}} \mathrm{Bu}\right) \mathrm{H}_{2}$ fällt außerhalb der Korrelation wegen intramolekularer $\mathrm{N}-\mathrm{H} \cdots \mathrm{N}$-Brücken [14]. 
Als z weiten Effekt findet man, daß beim Übergang ein und desselben Elementes von der Koordinationszahl 2 zur Koordinationszahl 3 (in den Kationen von $2 \mathrm{c}$ und $2 \mathrm{~d}$ werden $\mathrm{Sb}$ und $\mathrm{Bi}$ als quasi zweibindig betrachtet) der $\mathrm{N}-\mathrm{Si}-\mathrm{N}$-Winkel zunimmt bei fast gleichbleibendem oder leicht abnehmendem $\mathrm{Si}-\mathrm{N}$-Abstand. Dies bedeutet in allen Fällen eine „Hineinbewegung" des Siliciumatoms in Richtung der Ringmitte bei gleichzeitiger elektronischer „Kompensation“ am Element durch einen dritten Liganden. Hiervon ausgenommen ist die $\mathrm{Si}-\mathrm{N}$-Bindungslängenzunahme beim Übergang von $\mathrm{Me}_{2} \mathrm{Si}\left(\mathrm{N}^{t} \mathrm{Bu}\right)_{2} \mathrm{Sn}(5 \mathrm{e})$ zu

$\mathrm{Me}_{2} \mathrm{Si}\left(\mathrm{N}^{t} \mathrm{Bu}\right)_{2} \mathrm{Sn} \cdot \mathrm{N}\left(\mathrm{H}_{2}\right)^{t} \mathrm{Bu}$. Eine Erklärung für diese Ausnahme ist sehr leicht gefunden: im tert-Butylaminaddukt des Stannylens gibt es zusätzlich Wasserstoffbrückenbindungen zu den $\mathrm{N}$-Atomen des Vierringes, wodurch natürlich die $\mathrm{Si}-\mathrm{N}$-Bindung gelockert wird [14].

Aus den graphischen Auftragungen folgt für die formal zweifach koordinierten Elemente folgende Reihe zunehmender Wechselwirkung des Elementes mit den Stickstoffatomen:

$\mathrm{Me}_{2} \mathrm{Si}\left(\mathrm{N}^{\mathrm{t}} \mathrm{Bu}\right)_{2} \mathrm{Sn}<\mathrm{Me}_{2} \mathrm{Si}\left(\mathrm{N}^{\mathrm{t}} \mathrm{Bu}\right)_{2} \mathrm{Bi}^{+}<\mathrm{Me}_{2} \mathrm{Si}\left(\mathrm{N}^{\mathrm{t}} \mathrm{Bu}\right)_{2} \mathrm{Sb}^{+} \ll \mathrm{Me}_{2} \mathrm{Si}\left(\mathrm{N}^{t} \mathrm{Bu}\right) \mathrm{P}^{+}$.

Die Kompensation der Elektronenlücke am Element steigt in der gleichen Reihenfolge. Chemisch gesehen müssen die Kationen von $2 \mathrm{e}$ und $2 \mathrm{~d}$ ebenso wie $\mathrm{Me}_{2} \mathrm{Si}\left(\mathrm{N}^{\mathrm{t}} \mathrm{Bu}\right)_{2} \mathrm{Sn}$ starke Lewis-Säuren sein. Die im vorhergehenden Kapitel diskutierten Strukturen belegen diesen Befund für $2 \mathrm{e}$ und $\mathbf{2 d}$. Die chemische Relevanz dieser Aussage spiegelt sich im ehemischen Verhalten von $\mathrm{Me}_{2} \mathrm{Si}\left(\mathrm{N}^{t} \mathrm{Bu}\right)_{2} \mathrm{Sn}$ wider [24, 26]. Kontrastierend hierzu ist das Kation in 2a wenig elektrophil: in der Kristallstruktur findet man daher nur geringe Wechselwirkungen vom Donor-Akzeptor-Typ zwischen dem Anion und Kation.

\section{Experimenteller Teil}

Sämtliche Umsetzungen wurden unter Ausschluß von Luft und Feuchtigkeit unter Stickstoff als Schutzgas in Hochvakuumapparaturen durchgeführt. ${ }^{1} \mathrm{H}$-NMR-Spektren erhielten wir auf einem Bruker HFX 80, als Massenspektrometer diente ein Varian MAT CH-7 und als UV-Spektrometer ein SP800 Pye Unicam. Die C-, H-, N-Analysen führte die Firma O. Beller, Göttingen, aus.

1,3-Di-tert-butyl-2, 2-dimethyl-1,3,2,4 $\lambda^{2}$-diazasilaphosphonia-(a), -arsonia-(b), -stibonia(c),bismutonia-(d), tetrachloro-aluminat (2), -gallat (3), -indat (4)

Frisch sublimiertes Aluminium(III)-chlorid, Gallium(III)-chlorid oder Indium(III)-chlorid werden in Benzol gelöst (Ga) oder suspendiert (AI, In). Unter Rühren tropft man im molaren Unterschluß (Al, In) oder äquimolar eine Lösung des Bis(amino)elementchlorids 1a, 1b, 1c bzw. 1d hinzu [1, 2]. Hierbei beobachtet man eine Auflösung des Element(III)-chlorids und teilweise Farbänderung der Lösung. Nach kurzer Zeit fallen die Produkte feinkristallin aus. Nach Umkristallisieren aus Benzol oder 'Toluol erhält man $95 \%$ der theoretisch erwarteten Menge im Falle der Tetrachloroaluminate und -gallate, bzw. $60 \%$ im Falle der Tetrachloroindate. 1a setzt sich mit InCl $_{3}$ nicht zum gewünschten Produkt um und kann nach Entfernen des Lösungsmittels unzersetzt erhalten werden. 
$\mathbf{C}_{10} \mathbf{H}_{24} \mathbf{A l C l}_{4} \mathbf{N}_{2} \mathbf{P S i} \quad(400,17 \mathrm{~g} / \mathrm{mol})(2 \mathrm{a})$. Farblose Kristalle mit Schmelzpunkt: $118^{\circ} \mathrm{C}$ (Zers.). Massenspektrum: Gef. Peak höchster Masse m/e $=231$, ber. für $\mathrm{C}_{\mathbf{1 0}} \mathrm{H}_{\mathbf{2 4}} \mathrm{N}_{\mathbf{2}} \mathbf{P}^{28}$ Si (Kation von $\mathbf{2 a}$ ). ${ }^{1} \mathrm{H}-\mathrm{N}$ MR (Tetrahydrofuran): $\delta=0,5\left(1,0, \mathrm{~s} ; \mathrm{Si}-\mathrm{CH}_{3}\right), 1,4\left(3,0, \mathrm{~d}\left({ }^{4} \mathrm{~J}\left({ }^{\mathrm{I}} \mathrm{P}-{ }^{1} \mathrm{H}\right) 4 \mathrm{~Hz}\right) ; \mathrm{C}-\mathrm{CH}_{3}\right)$ ppm.

Ber. C 30,01, H 6,05, N 7,00, Cl 35,44; gef. C 29,90, H 5,88, N 7,02, Cl 35,40\%. $88^{\circ} \mathrm{C}$.

$\mathbf{C}_{10} \mathbf{H}_{24} \mathbf{A l A s C l}_{4} \mathbf{N}_{2} \mathbf{S i} \quad(444,17 \mathrm{~g} / \mathrm{mol})$, (2b). Nadelförmige, gelbe Kristalle mit Schmelzpunkt :

Massenspektrum: Gef. $\mathrm{m} / \mathrm{e}=275$, ber. für $\mathrm{C}_{10} \mathrm{H}_{24} \mathrm{AsN}_{2}{ }^{28} \mathrm{Si}$ (Kation von $\mathbf{2 b}$ ).

${ }^{1} \mathrm{H}-\mathrm{NMR}$ (Benzol): $\delta=0,11\left(1,0, \mathrm{~s} ; \mathrm{Si}-\mathrm{CH}_{3}\right), \mathbf{1 , 0 8}\left(3,0, \mathrm{~s} ; \mathrm{C}-\mathrm{CH}_{3}\right) \mathrm{ppm}$.

UV-Spektrum (Benzol) s. Tab. 1.

Ber. C 27,04, H 5,45, N 6,31, Cl 31,93, As 16,87, gef. C 27,08, H 5,55, N 6,28, Cl 31,31, As 17,3\%.

$\mathbf{C}_{10} \mathbf{H}_{24} \mathbf{A l C l}_{4} \mathbf{N}_{2} \mathbf{S b S i} \quad(491,00 \mathrm{~g} / \mathrm{mol})(\mathbf{2 e})$. Nadelförmige, gelbe Kristalle mit Schmelzpunkt: $123^{\circ} \mathrm{C}$.

Massenspektrum: Gef. $\mathrm{m} / \mathrm{e}=488$, ber. für $\mathrm{C}_{10} \mathrm{H}_{24} \mathrm{Al}^{35} \mathrm{Cl}_{4} \mathrm{~N}_{2}{ }^{121} \mathrm{Sb}^{28} \mathrm{Si} .{ }^{1} \mathrm{H}-\mathrm{NMR}$ (Benzol) : $\delta=0,13$ $\left(1,0, \mathrm{~s} ; \mathrm{Si}-\mathrm{CH}_{3}\right), 1,0\left(3,0, \mathrm{~s} ; \mathrm{C}-\mathrm{CH}_{3}\right)$ ppm. UV-Spektrum (in Benzol) s. Tab. 1.

Ber. C $24,46, \mathrm{H} 4,93, \mathrm{~N} 5,71$, Cl 28,88 , Sb 24,80 , gef. C 24,64 , H 5,09, N 5,74, Cl 27,79, Sb $24,68 \%$

$\mathbf{C}_{\mathbf{1 0}} \mathbf{H}_{\mathbf{2 4}} \mathbf{A l B i C l} \mathbf{N}_{\mathbf{2}} \mathbf{S i} \quad(578,23 \mathrm{~g} / \mathrm{mol})(\mathbf{2 d})$. Dunkelrote Kristalle mit Schmelzpunkt: $112^{\circ} \mathrm{C}$.

Molmasse in Benzol : gef.: $1068 \mathrm{~g} / \mathrm{mol}$ (dimeres Molekül!).

Massenspektrum: Gef.: $\mathrm{m} / \mathrm{e}=559$; ber. für $\mathrm{C}_{9} \mathrm{H}_{19} \mathrm{AlBi}^{35} \mathrm{Cl}_{4} \mathrm{~N}_{2}{ }^{28} \mathrm{Si}$.

${ }^{1} \mathrm{H}-\mathrm{NMR}$ (Benzol) : $\delta=0,08\left(1,0, \mathrm{~s} ; \mathrm{Si}-\mathrm{CH}_{3}\right), 1,0\left(3,0, \mathrm{~s} ; \mathrm{C}-\mathrm{CH}_{3}\right)$ ppm. UV-Spektrum (Benzol) s. Tab. 1.

Ber. C $20,77, \mathrm{H} 4,18, \mathrm{~N} 4,84, \mathrm{Cl} 24,53$, Bi 36,14, gef. C 20,90, H 4,30, N 4,74, Cl 24,35, Bi 34,6\%. $108^{\circ} \mathrm{C}$.

$\mathbf{C}_{10} \mathbf{H}_{24} \mathbf{C l}_{4} \mathbf{G a N}_{2} \mathbf{P S i} \quad(442,91 \mathrm{~g} / \mathrm{mol})(\mathbf{3 a})$. Farblose, nadelförmige Kristalle mit Schmelzpunkt:

${ }^{1} \mathrm{H}-\mathrm{NMR}$ (Benzol) $: \delta=0,27\left(1,0, \mathrm{~s} ; \mathrm{Si}-\mathrm{CH}_{3}\right), 1,10\left(3,0, \mathrm{~s} ; \mathrm{C}-\mathrm{CH}_{3}\right) \mathrm{ppm}$.

Ber. C 27,12, H 5,46, N 6,32, Cl 32,02, Ga 15,74, gef. C 26,41, H 5,53, N 5,60, Cl 28,68, Ga 15,32\%.

$\mathbf{C}_{\mathbf{1 0}} \mathbf{H}_{\mathbf{2 4}} \mathbf{A} \mathbf{s C l} \mathbf{G a N}_{2} \mathbf{S i} \quad(486,91 \mathrm{~g} / \mathrm{mol}) \quad \mathbf{( 3 \mathbf { b } )}$. Hellgelbe, nadelförmige Kristalle mit Schmelzpunkt: $95^{\circ} \mathrm{C}$.

Molmassenbestimmung in Benzol: gef. $479 \mathrm{~g} / \mathrm{mol}$.

Massenspektrum: Gef. m/e $=275$ ber. für $\mathrm{C}_{10} \mathrm{H}_{24} \mathrm{AsN}_{2}{ }^{28} \mathrm{Si}$ (Kation von $3 \mathrm{~b}$ ).

${ }^{1} \mathrm{H}-\mathrm{NMR}$ (Benzol) : $\delta=0,23\left(1,0, \mathrm{~s} ; \mathrm{Si}-\mathrm{CH}_{3}\right), 1,13\left(3,0, \mathrm{~s} ; \mathrm{C}-\mathrm{CH}_{3}\right)$ ppm. UV-Spektrum (Benzol) s. Tab. 1.

Ber. C 24,67, H 4,97, N 5,75, Cl 29,12, As 15,39, gef. C 24,27, H 5,14, N 5,54, Cl 28,13, As 15,18\%. $\mathbf{C}_{10} \mathbf{H}_{24} \mathbf{C l}_{4} \mathbf{G a N}_{2} \mathrm{SbSi} \quad(533,74)(3 \mathrm{c})$. Gelbe, nadelförmige Kristalle mit Schmelzpunkt: $143^{\circ} \mathrm{C}$. Molmassenbestimmung in Benzol: gef. $521 \mathrm{~g} / \mathrm{mol}$.

${ }^{1} \mathrm{H}-\mathrm{NMR}$ (Benzol) $\delta=0,08\left(1,0, \mathrm{~s} ; \mathrm{Si}-\mathrm{CH}_{3}\right), 1,02\left(3,0, \mathrm{~s} ; \mathrm{C}-\mathrm{CH}_{3}\right) \mathrm{ppm}$.

Ber. C 22,50, H 4,53, N 5,25, Cl 26,57, Sb 22,81, gef. C 22,51, H 4,60, N 5,20, Cl 26,39, Sb 22,95\%.

$\mathbf{C}_{10} \mathbf{H}_{24} \mathbf{B i C l}_{4} \mathbf{G a N}_{2} \mathbf{S i} \quad(620,97)(\mathbf{3 d})$. Rote Kristalle mit Schmelzpunkt: $94^{\circ} \mathrm{C}$.

Molmassenbestimmung in Benzol: $635 \mathrm{~g} / \mathrm{mol}$.

${ }^{1} \mathrm{H}-\mathrm{NMR}$ (Benzol) : $\delta=0,14\left(1,0, \mathrm{~s} ; \mathrm{SiCH}_{3}\right), 1,05\left(3,0, \mathrm{~s} ; \mathrm{C}-\mathrm{CH}_{3}\right)$ ppm.

Ber. C 19,34, H 3,90, N 4,51, Cl 22,84, Bi 33,65, gef. C 21,64, H 4,10, N 4,36, Cl 20,69, Bi 32,31\%.

$\mathbf{C}_{10} \mathbf{H}_{24} \mathbf{A s C l}_{4} \mathbf{I n N}_{2} \mathbf{S i} \quad(532,01)(\mathbf{4 b})$. Gelbe, nadelförmige Kristalle mit Schmelzpunkt: $139^{\circ} \mathrm{C}$. Massenspektrum: Gef. $\mathrm{m} / \mathrm{e}=275$, ber. für $\mathrm{C}_{10} \mathrm{H}_{24} \mathrm{AsN}_{2}{ }^{28} \mathrm{Si}$ (Kation von $4 \mathrm{~b}$ ).

UV-Spektrum (Benzol) s. Tab. 1.

${ }^{1} \mathrm{H}-\mathrm{NMR}$ (Benzol): $\delta=0,25\left(1,0, \mathrm{~s} ; \mathrm{Si}-\mathrm{CH}_{3}\right), 1,16\left(3,0, \mathrm{~s} ; \mathrm{C}-\mathrm{CH}_{3}\right) \mathrm{ppm}$.

Ber. C 22,58, H 4,55, N 5,27, Cl 26,66, As 14,08, gef. C 23,35, H 4,82, N 5,08, Cl 24,43, As 13,95\%.

$\mathbf{C}_{10} \mathbf{H}_{24} \mathbf{C l}_{4} \mathbf{I n N}_{2} \mathbf{S b S i} \quad(578,34)(\mathbf{4 c})$. Gelbe, quaderförmige Kristalle mit Schmelzpunkt: $118^{\circ} \mathrm{C}$.

${ }^{1} \mathrm{H}-\mathrm{NMR}$ (Benzol): $\delta=0,32\left(1,0, \mathrm{~s} ; \mathrm{Si}-\mathrm{CH}_{3}\right), 1,12\left(3,0, \mathrm{~s} ; \mathrm{C}-\mathrm{CH}_{3}\right)$ ppm.

Ber. C 20,75, H 4,18, N 4,84, Cl 24,50, Sb 21,03, gef. C 20,53, H 4,21, N 4,82, Cl 24,43, Sb 20,82\% 
$\mathbf{C}_{10} \mathbf{H}_{24} \mathbf{B i C l}_{4} \mathrm{InN}_{2} \mathrm{Si} \quad(666,07)(\mathbf{4 d})$. Dunkelrote Kristalle mit Sehmelzpunkt $120^{\circ} \mathrm{C}$.

${ }^{1} \mathrm{H}-\mathrm{NMR}$ (Benzol): $\delta=0,18\left(1,0, \mathrm{~s} ; \mathrm{Si}-\mathrm{CH}_{3}\right), \mathbf{1}, 15\left(3,0, \mathrm{~s} ; \mathrm{C}-\mathrm{CH}_{3}\right) \mathrm{ppm}$.

Ber. C 18,03, H 3,63, N 4,21, Cl 21,29, Bi 31,38, gef. C 18,68, H 3,70, N 4,20, Cl 18,78, Bi 31,64\%.

Rechnungen. Die Strukturrechnungen wurden am Rechenzentrum der TU Braunschweig bzw. am Rechenzentrum der Universität des Saarlandes mit Hilfe des SHELX-Systems [28] und des MULTAN- und ORTEP-Programms [29, 30] durchgeführt.

Dem Fonds der Chemischen Industrie danken wir für Hilfen bei Teilen dieses Vorhabens.

\section{Literatur}

[1] Veith, M.; Bertsch, B.: Z. anorg. allg. Chem. 557 (1988) 7.

[2] Scherer, O.J.; Püttmann, M.; Krüger, C.; Wolmershäuser, G.: Chem. Ber. 115 (1982) 2076.

[3] Veith, M.; Grosser, M.; HuCh, V.: Z. anorg. allg. Chem. 513 (1984) 89.

[4] Veiтh, M.: Angew. Chem. 87 (1975) 287; Angew. Chem. Int. Ed. Engl. 14 (1975) 263.

[5] Vetth, M.; Grosser, M.: Z. Naturforsch. 37b (1982) 1375.

[6] Kopp, R. W.; Bond, A. C.; PARry, R. W.: Inorg. Chem. 15 (1976) 3042.

[7] Thomas, M. G.; Solultze, C. W.; Parkx, R. W.: Inorg. Chem. 16 (1977) 994.

[8] Cowley, A. H.; Cushner, M. C.; Szobota, J. S.: J. Amer. Chem. Soc. 100 (1978) 7784.

[9] Cowley, A. H.; Cushner, M. C.; Latmann, M.; McKee, M. I.; Szobota, J. S.; Wilblre, J. C.: Pure Appl. Chem. 52 (1980) 789.

[10] Cowley, A. H.; Lattman, M.; Whburn, J. C.: Inorg. Chem. 20 (1981) 2916.

[11] Flemming, S.; Lupton, M. K.; Jекот, K.: Inorg. Chem. 11 (1972) 2534.

[12] Nrecke, E.; Kröher, R.: Angew. Chem. 88 (1976) 22.

[13] Cowlex, A. H.; KenP, R. A.: Chem. Rev. 85 (1985) 367.

[14] Veiтh, M.; Schlenmer, G.; Sommer, M.-L.: Z. anorg. allg. Chem. 497 (1983) 157.

[15] HuCH, V.: Dissertation, TU Braunschweig, 1984.

[16] Bertsch, B.: Dissertation, Universität des Saarlandes, Saarbrücken, 1986.

[17] Huheey, J. E.: Tnorganic Chemistry, $3^{\text {rd }}$ ed., Harper \& Row, New York 1983.

[18] Poulsen, F. R.; Rasmussen, S. E.: Acta Chem. Scand. 24 (1970) 150.

[19] Verreh, M.; BäRnighausen, H.: Acta Cryst. B30 (1974) 1806.

[20] VeITH, M.; HvCH, V.: J. Organomet. Chem. 293 (1985) 161.

[21] Niecke, E.; RüGer, R.; Lysek, M.; Pohl, S.; Schoeller, W.: Angew. Chem. 95 (1983) 495 Angew. Chem. Int. Ed. Engl. 2Q (1983) 486 und hierin zitierte Literatur.

[22] Baenzinger, H. C.: Acta Crystallogr. 4 (1951) 216.

[23] WeLts, A. F.: Structural Inorg. Chem., $5^{\text {th }}$ ed., Clarendon, Oxford 1984, S. $846 \mathrm{f}$.

[24] VeIтн, M.: Angew. Chem. 99 (1987) 1; Angew. Chem. Int. Ed. Engl. 26 (1987) 1.

[25] Vеттн, M.: Z. Naturforsch. 33b (1978) 7.

[26] VeIth, M.: J. Organomet. Chem. Libr. 12 (1981) 319.

[27] Hamiton, W. C.: Acta Crystallogr. 12 (1959) 609.

[28] Sheldrick, G.: Program for Crystal Structure Determination, Version SHELX-76.

[29] Matn, P.; Hull, S.; Lessinger, L.; Germatn, G.; Declerrq, J. P.; Woolfson, M. M. : MUlTaN, England 1978.

[30] Johrson, C. K.: ORTEP, ORNL-3794, Oak Ridge National Laboratory, 1965.

Bei der Redaktion eingegangen am 8. Juli 1987.

Anschr. d. Verf.: Prof. Dr. M. Veith, Dr. B. Bertsch, Dr. V. Huch, Fachbereich 13, Inst. f. Anorg. Chemie d. Univ., D-6600 Saarbrücken 